\title{
Anatomy of footprint extension in ribosome profiling reveals translational landscape mediated by $\mathbf{S 1}$ protein in bacteria
}

\section{Tomoya Fujita}

Tokyo Institute of Technology

\section{Takeshi Yokoyama}

RIKEN

\section{Mikako Shirouzu}

RIKEN Center for Biosystems Dynamics Research https://orcid.org/0000-0002-7997-2149

\section{Hideki Taguchi}

Tokyo Institute of Technology https://orcid.org/0000-0002-6612-9339

\section{Takuhiro Ito}

RIKEN https://orcid.org/0000-0003-3704-5205

Shintaro Iwasaki ( $\nabla$ shintaro.iwasaki@riken.jp )

RIKEN https://orcid.org/0000-0001-7724-3754

\section{Article}

Keywords: mRNA, RNasae footprinting, ribosome profiling, S1 protein

Posted Date: July 29th, 2020

DOl: https://doi.org/10.21203/rs.3.rs-41219/v1

License: (1) This work is licensed under a Creative Commons Attribution 4.0 International License. Read Full License 


\title{
Anatomy of footprint extension in ribosome profiling reveals translational landscape mediated by $\mathrm{S} 1$ protein in bacteria
}

\author{
Tomoya Fujita ${ }^{1,2}$, Takeshi Yokoyama ${ }^{3,4}$, Mikako Shirouzu ${ }^{3}$, Hideki Taguchi ${ }^{2,5}$, \\ Takuhiro Ito ${ }^{6}$, and Shintaro Iwasaki ${ }^{1,7,8 \dagger}$
}

${ }^{1}$ RNA Systems Biochemistry Laboratory, RIKEN Cluster for Pioneering Research, Wako, Saitama 351-0198 Japan

${ }^{2}$ School of Life Science and Technology, Tokyo Institute of Technology, Midori-ku, Yokohama 226-8503, Japan

${ }^{3}$ Laboratory for Protein Functional and Structural Biology, RIKEN Center for Biosystems Dynamics Research, Tsurumi-ku, Yokohama 230-0045, Japan

${ }^{4}$ Graduate School of Life Sciences, Tohoku University, 2-1-1 Katahira, Aoba-ku, Sendai 980-8577, Japan

${ }^{5}$ Cell Biology Center, Institute of Innovative Research, Tokyo Institute of Technology, Yokohama, Midori-ku, Yokohama 226-8503, Japan

${ }^{6}$ Laboratory for Translation Structural Biology, RIKEN Center for Biosystems Dynamics Research, Tsurumi-ku, Yokohama 230-0045, Japan

${ }^{7}$ Department of Computational Biology and Medical Sciences, Graduate School of Frontier Sciences, The University of Tokyo, Kashiwa, Chiba 277-8561, Japan

${ }^{8}$ Lead contact

${ }^{\dagger}$ Correspondence should be addressed to S.I.

E-mail: shintaro.iwasaki@riken.jp 


\section{$\underline{\text { Summary }}$}

Ribosome profiling - RNase footprinting of ribosome-bound mRNA - has been a unique and powerful method, applied to widespread organisms to survey ribosome traversal along mRNAs. In contrast to eukaryotes, bacterial ribosome footprints show a broad range of sizes, reflecting the differential states of ribosomes. However, the origin remains unclear. Here, we show that rotated state of ribosomes and intramolecular RNA duplexes each extend bacterial ribosome footprints at the 5' end. Combining elongation inhibitors, cryo-electron microscopy, and ribosome profiling, we demonstrated that the rotated state of ribosomes results in long footprints. Along the subunit rotation, ribosomal protein $\mathrm{S} 1$ - a 30S-subunit RNAbinding protein - sterically protects $\mathrm{mRNA}$ at the $5^{\prime}$ end of the ribosome from RNase digestion and facilitates elongation of the ribosome. Moreover, we found that ribosomes stalled on $y c b Z$ mRNA generate prolonged footprints because of their unique RNA secondary structure proximal to ribosomes. Through the studies of footprint extension, our results revealed S1-mediated stabilization of translation elongation and provide ribosome profiling approach to probe the conformational diversity of ribosomes in bacteria. 


\section{$\underline{\text { Introduction }}$}

Ribosomes traverse along mRNAs to decode codons and synthesize amino acid chains (Voorhees and Ramakrishnan, 2013). This translation elongation is accompanied with tRNA translocation in the inter subunit cavity driven by global conformational rearrangements of the $70 \mathrm{~S}$ ribosome, such as a rotation of two subunits (Voorhees and Ramakrishnan, 2013). In the non-rotated state, ribosomes possess peptidyl-tRNA at the $\mathrm{P}$ site and the codon at the vacant A site undergoes the initial selection of the appropriate aminoacyl-tRNA with the aid of EF-Tu. After the peptidyl transfer reaction, the small subunit (30S) rotates relative to the large subunit (50S) in a counterclockwise manner (i.e., rotated state) with the guide of EF-G and then dynamic positional rearrangement of tRNAs is induced: tRNAs in ribosomes occupy hybrid $\mathrm{E} / \mathrm{P}$ and $\mathrm{P} / \mathrm{A}$ positions, in which P- and A-site tRNA heads lean toward the E and P sites of the 50S subunit, respectively. Finally, the translocation of ribosomes is prompted by GTP hydrolysis of EF-G, and then the ribosome returns to a non-rotated state for the next round of the elongation cycle. This elongation process is even more complicated in cells; various factors (e.g., tRNA availabilities, RNA secondary structures, and nascent peptide-ribosome exit tunnel interaction) halt the elongation cycle in the middle and stall ribosomes on mRNAs (Nakatogawa and Ito, 2002; Lu and Deutsch, 2008; Yanagitani et al., 2011; Dana and Tuller, 2012; Brandman et al., 2012; Charneski and Hurst, 2013; Pop et al., 2014; Hussmann et al., 2015; Weinberg et al., 2016; Zhang et al., 2017; Dao Duc and Song, 2018; Ibrahim et al., 2018; Diament et al., 2018; Mohammad et al., 2019; Sharma et al., 2019).

Ribosome profiling has been proven to be a powerful and sensitive tool to measure ribosome movement along mRNA in vivo (Ingolia et al., 2009; McGlincy and 
Ingolia, 2017; Iwasaki and Ingolia, 2017). Harnessing the protection of RNA fragments by ribosomes from RNase digestion and sequencing of the "ribosome footprints" allows us to survey ribosome occupancy across the transcriptome. Since RNase accessibility determines the resultant size of the RNA fragment, it is reasonable to suppose that the conformational rearrangements in different ribosomal states affect the footprint lengths. While eukaryotic ribosomes generate two distinct footprint sizes depending upon A-site tRNA occupancy (Lareau et al., 2014; Matsuo et al., 2017; Kurihara et al., 2018; Wu et al., 2019), E. coli ribosomes have been reported to have a broad footprint size range (Mohammad et al., 2016; Mohammad et al., 2019). However, the rationales of the origin of widely distributed footprint size and its relevance to ribosome states remain unknown.

Here, we show that $5^{\prime}$-extended, long footprints highly correlated to the rotated state of ribosomes in bacteria. Testing a series of translation inhibitors in ribosome profiling, we found that ribosomes trapped in the rotated state result in 35-40 nt footprints, while non-rotated ribosomes generate 23-28 nt footprints. Ribosomal protein S1, which composes the small ribosomal subunit and is located close to the mRNA exit channel, contributed to footprint extension toward the $5^{\prime}$ end. The interaction between $\mathrm{S} 1$ and mRNA stabilized elongation step and facilitated protein synthesis from long coding sequences (CDS). In addition, we found that a subset of codon positions still retained long footprints regardless of drug treatment. A ribosome pause site on $y c b Z$ mRNA coincided with highly structured RNA docked to ribosomes, rendering footprints resistant to RNase treatment. This work provides an insight into stabilization mechanism of rotating ribosomes during elongation reactions and a framework to link ribosome footprint size and ribosome states in bacteria. 


\section{$\underline{\text { Results }}$}

\section{Rotated state of ribosomes results in longer ribosome-protected fragments}

Given the divergent footprint length reported in bacteria (Mohammad et al., 2016; Mohammad et al., 2019), we reasoned that conformational differences in ribosomes may confer differential RNase sensitivity. To trap ribosomes in different states during elongation cycles, we employed a variety of translation inhibitors for ribosome profiling in E. coli: chloramphenicol (Bulkley et al., 2010; Dunkle et al., 2010), linezolid (Wilson et al., 2008), blasticidin (Svidritskiy et al., 2013), capreomycin (Stanley et al., 2010), viomycin (Ermolenko et al., 2007; Cornish et al., 2008; Stanley et al., 2010; Wang et al.,

2012), and spectinomycin (Carter et al., 2000; Borovinskaya et al., 2007). To avoid possible artefacts caused by drug pre-treatment in cell culture (Ingolia et al., 2012; Hussmann et al., 2015) and fragment size selection (Mohammad et al., 2016), we treated the samples with the drugs in cell lysis and covered a wide range of footprint sizes (17$50 \mathrm{nt})$ during the library preparation.

Remarkably, the drugs could be sub-classified into two groups based on the footprint size distribution. The first group of inhibitors, including chloramphenicol (a standard for bacterial ribosome profiling), linezolid, and blasticidin, showed that the footprints peaked at $\sim 28 \mathrm{nt}$, as observed in a drug-free condition (Fig. 1a). This size has been observed in earlier works (Mohammad et al., 2016; Mohammad et al., 2019). In contrast, the second group of inhibitors - capreomycin, viomycin, and spectinomycin — extended ribosome footprints toward 35-43 nt (Fig. 1b).

Indeed, the sub-classification corresponded to the ribosome conformations trapped by the drugs. The first group of inhibitors (chloramphenicol, linezolid, and blasticidin) binds to the peptidyl transferase center (PTC) of the large subunit (50S), 
blocks peptide bond formation, and thus stabilizes ribosomes in a non-rotated state (Wilson et al., 2008; Bulkley et al., 2010; Dunkle et al., 2010; Svidritskiy et al., 2013). The second group of inhibitors, capreomycin and viomycin are known to bind to the 16S rRNA decoding center located on the 30S-subunit A site, thereby inhibiting the translocation of mRNA and tRNAs (Ermolenko et al., 2007; Cornish et al., 2008; Stanley et al., 2010; Dunkle et al., 2011; Wang et al., 2012). Spectinomycin binds to the head domain of the 30S small subunit (Borovinskaya et al., 2007; Carter et al., 2000) to block head swiveling after subunit rotation. The dose-dependent effect of the second group of drugs further supported that the prolonged footprints were due to compound treatment (Supplementary Fig. 1a-c). Although footprint extension was observed for all the second group of inhibitors, the weaker effects of spectinomycin might originate from the mechanistic difference in drugs to halt ribosomes in the rotated state.

To further characterize the longer footprints, we categorized the reads obtained into two bins based on the read length (short; 23-28 nt and long; 35-40 nt). Meta-gene analysis confirmed the enrichment of long footprints along the CDS in capreomycin treatment compared with chloramphenicol treatment (Fig.1c, d). Moreover, the 3-nt periodicity of footprints (Fig. 1c, d) provided further evidence that the longer footprints emerged from elongating ribosomes, as observed in regular short footprints. The high reproducibility of footprints on each CDS showed that capreomycin treatment did not bias ribosome footprints toward specific mRNAs (Supplementary Fig. 1d). Similarly, the drug did not alter ribosome occupancy on each codon species (Supplementary Fig. 1e). Thus, capreomycin extended footprints from elongating ribosomes throughout codons across the transcriptome. 
Since the earlier structural basis of capreomycin action has been restricted in Thermus thermophilus 70S (Stanley et al., 2010), we examined whether the drugs trap ribosome conformations in E. coli by cryo-electron microscopy single particle analysis (cryo-EM) (Fig. 1e-h, Supplementary Fig. 2a-g, i, j, and Supplementary Table 1). The three-dimensional classification based on the global conformation of the $70 \mathrm{~S}$ ribosome showed that the majority (52\%) of the E. coli ribosomes treated with capreomycin were populated in the rotated state with two tRNAs at hybrid positions (P/E-tRNA and A/PtRNA) (Fig. 1f and Supplementary Fig. 2b). Notably, the distinct mass of cryo-EM density attributed for capreomycin was clearly observed at the 16S rRNA decoding center (Fig. 1h). The sable binding of capreomycin flips A1492 and A1493 bases, which maintain fidelity of decoding. This observation is consistent with earlier observations in Thermus thermophilus (Stanley et al., 2010). Predictably, in the chloramphenicol-treated sample, the major population of particles converged to non-rotated ribosomes with chloramphenicol bindings (Fig. 1e, g, and Supplementary Fig. 2a). We also observed unpredicted rotated state of ribosomes with P/E tRNA (Supplementary Fig. 2a, i, j), accompanied with A/P tRNA, which was barely observed as fragmented cryo-EM densities in the lower contour level. This is feasible that chloramphenicol binds to the PTC after tRNAs' leaning and subunit rotation, although we suspect this situation is not frequent. Taken together, the ribosome conformations confined by the drugs led to the differential footprint size in ribosome profiling.

Conformational annotations of characterized ribosome pause sites by ribosome profiling 
The structure-footprint size correlation allowed us to address ribosome conformations at a sub-codon resolution. Here we revisited to the reported translation pause sites. To investigate the naïve ribosome conformation, ribosome profiling without drug treatment was analysed. The tnaC pause site at the stop codon is an example: two L-Trp molecules bind to the pocket formed between the ribosome exit tunnel and the nascent chain, position PTC in an incompatible conformation, and then trap the ribosome in a nonrotated state (Gong et al., 2001; Bischoff et al., 2014). As expected in the structural study, we observed the prominent accumulation of short footprints at the corresponding pause site (Fig. 1i).

The second example of ribosome pauses focuses on secM, decoding Pro166 at the A site (Nakatogawa and Ito, 2001; Nakatogawa and Ito, 2002; Muto et al., 2006; Zhang et al., 2015). This nascent chain upstream of the pause site interacts with the ribosome exit tunnel and eventually induces two different consequences in the structure to be stalled (Zhang et al., 2015): inactivation of the peptidyl transfer reaction and long duration of peptidyl-tRNA at the A/P hybrid position. As a result, ribosomes are paused in both non-rotated and rotated states (Zhang et al., 2015). Concordant with the structural analysis, ribosomes on the $\sec M$ pause site accumulated both long and short footprints (Fig. 1j). Taken together, the characterization of ribosome pause sites further supported the categorization of rotated and non-rotated states by footprint lengths.

\section{Long footprints are extended at the $5^{\prime}$ end}

We were intrigued by the molecular basis of the footprint extension. To investigate the relative direction of extension on footprints, we calculated ribosome occupancies around 
stop codons which enrich the footprints in general, assigning the $5^{\prime}$ and $3^{\prime}$ ends of the ribosome footprints. The ribosome under termination or recycling was denoted by a region between the peaks in $5^{\prime}$ and $3^{\prime}$ assigning that locates upstream and downstream of stop codon, respectively (Fig. 2a). Whereas the $3^{\prime}$ ends of footprints were at the same position in short and long footprints, the $5^{\prime}$ ends were shifted along the read extension (Fig. 2a). Thus, the major difference in footprint length originated from variable $5^{\prime}$ ends. Similar observations were reported previously in bacterial ribosome footprints (Woolstenhulme et al., 2015; Mohammad et al., 2016; Mohammad et al., 2019).

Ribosomal protein $S 1$ protects the $5^{\prime}$ end of ribosome footprints in rotated state of ribosomes

Given the variable $5^{\prime}$ ends, we reasoned that factor(s) close to the $5^{\prime}$ ends of ribosomes may affect RNase accessibility. An apparent candidate is ribosomal protein S1 (S1), which lies adjacent to the mRNA exit channel in the 30S subunit (Byrgazov et al., 2015; Demo et al., 2017; Beckert et al., 2018). S1 is an RNA binding protein and consists of six repeats of OB-fold domains. The first two OB-fold repeats directly interact with $30 \mathrm{~S}$ (Byrgazov et al., 2015), and the subsequent four domains can bind to mRNA (Boni et al., 1991; Sengupta et al., 2001; Qu et al., 2012; Duval et al., 2013), covering a 10-nt-long region (Qu et al., 2012)

To test the contribution of S1 to footprint extension, we attenuated the RNA binding ability of the protein. Although S1 could not be depleted from cells because of its essential role in general translation, the partial deletion of the RNA binding region was feasible (S1 $\Delta 56$ strain) (Duval et al., 2013) (Supplementary Fig. 3a, b). Ribosome profiling from 
the strain with truncated S1 showed reduced long footprints with capreomycin (Fig. 2c). On the other hand, the short footprints generated by chloramphenicol treatment were not affected by mutated S1 (Fig. 2b). Thus, these data indicated that S1 prolongs the footprints in a ribosome conformation-specific manner.

$\mathrm{S} 1$ has been proposed to function in translation initiation in a form of $30 \mathrm{~S}$; S1 directly associates with the U-rich region of mRNA on the upstream of the start codon, recruits the 30S subunit to mRNA, and facilitates the recognition of the start codon (Duval et al., 2013). In contrast, S1's effect on ribosome footprint extension suggested that S1 also associates with mRNA on the elongating $70 \mathrm{~S}$ ribosome. To examine this scenario, we monitored the position of $\mathrm{S} 1$ along transcripts by UV-C crosslinking immunoprecipitation sequencing with an infrared labeled linker (irCLIP-seq) (Zarnegar et al., 2016) (Supplementary Fig. 3c). As expected by the role of S1 in translation initiation, we observed the pile-up of the reads upstream of initiation codons (Supplementary Fig. 3d). The peak position ( $20 \mathrm{nt}$ upstream of the start codon) was consistent with the location of S1 on the 30S ribosome (Boni et al., 1991). In addition to the 5' UTR, the irCLIP-seq reads were also found in the CDS (Supplementary Fig. 3d), providing evidence of the interaction of S1 with mRNA in the elongating 70S.

Indeed, the enrichments of irCLIP-seq reads by capreomycin treatment around the stop codon corresponded closely to the footprint extension by the drug (Fig. 2d). Thus, the data suggested that, in the rotated state, $\mathrm{S} 1$ on the $70 \mathrm{~S}$ ribosome has a higher chance of associating with the mRNA region close to the mRNA exit channel. It is most likely that the S1 interaction sterically withstands RNase treatment and thus extends the footprints at the $5^{\prime}$ ends. 


\section{S1 stabilizes elongation ribosomes and facilitates protein synthesis from long CDS.}

Given that S1 interacts with mRNA in CDS (Supplementary Fig. 3d), we reasoned that S1 may have a role in translation elongation in addition to translation initiation. Indeed, calculating footprint distribution on each CDS [i.e. polarity score (Schuller et al., 2017)], we found that S1 $\Delta 56$ strain biased the footprint toward 5' direction, especially in long CDS (300 codons or longer) (Fig. 3a). These data suggested that elongation in S1 $\Delta 56$ strain was delayed or aborted.

To test this possibility, we set out in vitro translation with S1-depleted ribosomes. To avoid the effect of S1 depletion on translation initiation, we designed the firefly luciferase reporter fused to $\operatorname{sodB} 5^{\prime}$ UTR, which allows S1-independent initiation (Duval et al., 2013) (Fig. 3b, top). The extension of CDS with single or tandem GFP sequences in reporter reduced protein synthesis driven by S1-depleted ribosomes (Fig. 3b, bottom), as a readout of elongation defects. The supplementation of recombinant S1 protein (Supplementary Fig. 3e) rescued the translation reactions (Fig. 3b, bottom). We note that S1 depletion per se did not strongly affect the translation in our condition,

probably because S1 was dropped off ribosomes during preparation and thus at substoichiometric in in vitro translation system (PURE system) (Supplementary Fig. 3e) as reported in another ribosome protein (Chadani et al., 2017). Taken together, these data suggested that $\mathrm{S} 1$ facilitates translation elongation, associating with mRNAs during rotation of ribosomes.

Footprint extension beyond ribosome rotation

Fujita et al. p11 
Although we found that the inter-subunit rotation of ribosomes contributes to differential footprint sizes, a fraction of extended footprints could be attributed to other rationales. For example, duplex formation between the Shine-Dalgarno (SD) sequence in mRNA and the anti-SD sequence in 16S rRNA, which is docked in the chamber on $30 \mathrm{~S}$ (Kaminishi et al., 2007) was reported to block trimming by RNase (Mohammad et al., 2016; Mohammad et al., 2019). Our ribosome profiling also showed that the SD-like Grich sequence (e.g., UGG) resulted in longer footprint accumulation $\sim 20-25 \mathrm{nt}$ downstream (Supplementary Fig. 4a), where the anti-SD sequence of 16S rRNA is located. Such extension was minor overall but was still found in a fraction of ribosome footprints in the chloramphenicol-treated ribosome profiling library. Indeed, the regression analysis of two Gaussian distribution formulas modeled two populations, which consisted of a major population peak at $\sim 25 \mathrm{nt}$ and a minor population peak at $\sim 35$ nt (Supplementary Fig. 4b).

To identify a factor altering the footprint length beyond the inter-subunit rotation, we surveyed a specific codon position biased toward long footprints across the transcriptome irrespective of drug treatment. Considering footprint sizes, we calculated the ribosome pause score, which is the ratio of reads to average reads per codon at the given mRNA (Schuller et al., 2017), at the A-site codon under drug-free conditions (Fig. 4a). Here, we defined 64 codons in which long footprints were over-represented compared with short footprints (Fig. 4a). Many of these still possessed long footprints even after chloramphenicol treatment (Fig. 4b), which should convert footprints to short footprints. Consistent with the footprint extension by the SD-like sequence, a fraction of the long-footprint-biased pause sites possessed a low free energy $(\Delta \mathrm{G})$ between the antiSD sequence on 16S rRNA and the sequence upstream of the pause site (Supplementary 
Fig. 4c, e.g., lower than red dot line). However, a subset of the codons (such as $y c b Z$ pause site, see below for the details) still could not be explained by the SD-like sequence (Supplementary Fig. 4c, e.g., higher than top black dot line), implying other molecular bases for footprint extension.

\section{Ribosomes are stalled on ycbZ}

Then, we characterized the codon sites accompanied by drug-independent long footprints. Because we considered the over-accumulation of long footprints in the survey of codon positions, the sites naturally had higher footprints overall and thus are candidates for ribosome pause sites. To test the stalling of ribosomes on those sites, we performed in vitro integrated nascent chain profiling (iNP) (Chadani et al., 2016) using a reconstituted cell-free translation system (Shimizu et al., 2001) with ${ }^{35}$ S methionine. In this experiment, the accumulation of intermediate peptidyl-tRNA, which migrates slowly in SDS-PAGE at neutral $\mathrm{pH}$ because of tRNA $(\sim 20 \mathrm{kDa})$ conjugation, was used as a readout of ribosome pause. Along with well-characterized $s e c M, 6$ out of 9 selected mRNAs with longer footprint pause sites - ispH, napD, nuoA, sseA, $y c b Z, y k g F$, hybA, hybB, and $l p x C$ (Fig. $4 a, b)$ - led to high molecular weight products (Fig. 4c, d). The disappearance of the signals after RNase treatments validated the presence of a tRNA moiety on the peptide (Fig. 4c).

Among the pause sites with long footprints, we focused the site found in $y c b Z$ just before the stop codon (Fig. 5a and Supplementary Fig. 5a), since iNP on the mRNA provided strongest peptidyl-tRNA accumulation among the tested mRNAs. The presence of the footprint of the disome (Subramaniam et al., 2014) — two ribosomes colliding in 
a unit — on the exact same site (Pro585 at the P site and Asn586 at the A site) further supported that the elongation reaction on the $y c b Z$ transcript is slow enough for trailing ribosomes to catch up to the leading ribosome (Fig. 5b and Supplementary Fig. 5b). To uncover the minimum region critical for the pause, we designed a reporter for in vitro translation. Given that the amino acid/RNA contexts around the pause sites have been reported to impede translation elongation (Nakatogawa and Ito, 2002; Lu and Deutsch, 2008; Yanagitani et al., 2011; Dana and Tuller, 2012; Brandman et al., 2012; Charneski and Hurst, 2013; Weinberg et al., 2016; Dao Duc and Song, 2018), we placed the 30 amino acids upstream from the pause site, which ranges from the A site to the end of the nascent chain held within the ribosome exit tunnel, into a reporter (Fig. 5c, top and Supplementary Fig. 5c). The translated products were detected by Western blotting with a HA-tag inserted in front of the pause site. Indeed, the inserted segment of $y c b Z$ was sufficient to drive RNase-sensitive peptidyl-tRNA from the reporter (Fig. 5c, bottom). The substitution of the 30 amino acid sequence into the unrelated tag sequence (streptavidin binding protein or SBP) reduced the pause (Supplementary Fig. 5d).

We further narrowed down the critical amino acid for ribosome pause. Taking the amino acid sequence conservation among species (e.g., Vibrio, Yersinia, Klebsiella, and Salmonella) (Supplementary Fig. 5c) and residues immediately upstream of the pause site into account, we performed alanine-scanning mutagenesis. We found that Asn586, which is assumed to correspond to the A site of the paused ribosome, is crucial for pausing translation (Supplementary Fig. 4d).

To gain evidence of ribosome pause at the expected site, we investigated the tRNAs held in the stalled ribosome. The ribosome-nascent chain complex (RNC) on the pause site was affinity-purified via His-tag on the nascent peptide, followed by a sucrose 
density gradient (Supplementary Fig. 5e-g). Sequencing of the tRNAs within the complex revealed that tRNA ${ }^{\mathrm{Asn}}{ }_{\mathrm{GUU}}$ and $\mathrm{tRNA}{ }^{\mathrm{Pro}}{ }_{\mathrm{CGG}}$, whose anti-codons correspond to the $\mathrm{A}$ and $\mathrm{P}$ sites of codons of the pause site, were enriched in the RNC (Fig. 5d).

Direct visualization of the stalled ribosome by single particle cryo-EM further supported the assignment of the paused codon. The structural features of the cryo-EM density corresponding to P-site tRNA in the non-rotated state 70S was compatible with peptidyl-tRNA ${ }^{\text {Pro }}{ }_{\mathrm{CGG}}$ (Fig. 5e, f), which was consistent with ribosome profiling, tRNAseq, and biochemical analysis (Fig. 4). Thus, we concluded that the ribosomes translating $y c b Z$ mRNA were stalled on Pro585-Asn586 at their P-A sites (Fig. 5a, b).

\section{mRNA secondary structure alters RNase accessibility}

During the cryo-EM analysis of the stalled ribosomes on $y c b Z$ mRNA, we could not find any rational structural feature to explain footprint extension in ribosome profiling; the ribosome was non-rotated state with P-site tRNA. Thus, we reasoned that, rather than ribosomes, $y c b Z$ mRNA has an element that alters RNase accessibility and that, analogous to the SD/anti-SD duplex, the secondary structure of mRNA may be such a block. High base pairing of $y c b Z$ mRNAs upstream of the pause site was observed in DMS-seq (Burkhardt et al., 2017) (Fig. 5g). In addition, RNA secondary structure prediction by CentroidFold (Sato et al., 2009) indicated base pairing in footprint extended region (Supplementary Fig. 6a). Similar duplex formation was also found in other sites with drug-independent long footprints (Supplementary Fig. 6a, b). Taken together, our results showed the diverse origins of footprint extension, primarily ribosome subunit rotation and secondarily mRNA secondary structure (Fig. 6). 


\section{$\underline{\text { Discussion }}$}

Since development of ribosome profiling (Ingolia et al., 2009), the method has been used to address three major issues in cells: translation efficiency (over- or under-representation of footprint counts vs. RNA-Seq on CDS), coding region assignment, and elongation speed (Ingolia, 2014; Brar and Weissman, 2015; Fujita et al., 2019). In addition, our work indicated that by focusing on footprint length, ribosome profiling allows us to discuss the state of ribosome rotation during elongation in cells.

In eukaryotes, ribosome footprints show two distinct populations with peaking at sizes of $\sim 22 \mathrm{nt}$ and $\sim 29 \mathrm{nt}$ (Lareau et al., 2014; Matsuo et al., 2017; Kurihara et al., 2018; $\mathrm{Wu}$ et al., 2019). Indeed, a recent study reported that the footprint sizes represent differential RNase accessibility at the $3^{\prime}$ end originating from the accommodation of tRNA at the A site: the tRNA extends the footprint toward the $3^{\prime}$ end of footprints (Wu et al., 2019). In contrast, the bacterial ribosome footprint may not be reflected by A-site tRNA since the extended direction is the $5^{\prime}$ side rather than the $3^{\prime}$ end. Thus, the direction and molecular basis of footprint extension are essentially disparate in prokaryotes and eukaryotes. Notably, ribosomal protein S1 is found only in prokaryotes; thus, $5^{\prime}$ end variability could not occur in eukaryotes.

As shown in this study, the footprint length is directly associated with the rotation of ribosomal subunits in E. coli. In eukaryotic ribosome footprints, this is a challenge since the pre-peptidyl transfer state (non-rotated ribosome with tRNA at the A site) and pre-translocation state (rotated ribosome with leaned tRNA at the A/P site) can both form long footprints and thus could not be distinguished. However, the cocktail of cycloheximide and tigecycline, which inhibits tRNA translocation from the P site to the E site (Schneider-Poetsch et al., 2010; Garreau de Loubresse et al., 2014) and A-site 
tRNA accommodation (Jenner et al., 2013), respectively, allows minimization of the fraction of pre-peptidyl transfer (non-rotated) states and eventually increases the pretranslocation state (rotated) ribosome fraction in long footprints (Wu et al., 2019).

During the study of ribosome pauses with long footprints, we found that translation paused on the CDS of $y c b Z$. The current cryo-EM structure of the paused ribosomes could not determine the origin of the slowdown of the elongation cycle at this site, possibly because of the averaged view of multiple states of the isolated ribosomes. Moreover, the clear drop-off of A-site Asn-tRNA, which was assessed by tRNA-seq, also hampered our understanding. Although the YcbZ remains uncharacterized, its role in the suppression of stop codon readthrough was suggested (Gagarinova et al., 2016). Future studies regarding the molecular function of the YcbZ protein and the role of pause during its synthesis are warranted.

In conclusion, the states of ribosomes during the elongation cycle revealed by footprint length provide a useful framework and resource to identify the translational landscape in bacteria. 


\section{$\underline{\text { Acknowledgements }}$}

We are grateful to all the members of the Iwasaki and Taguchi laboratories for constructive discussion, technical help, and critical reading of the manuscript. We also thank Yuhei Chadani for technical advice to iNP, Christian Spahn and Hiroshi Yamamoto for the anti-S1 antibody, Stefano Marzi for the $\operatorname{rps} A 1, \operatorname{rps} A \Delta 5$, and $\operatorname{rps} A \Delta 56$ strains, and Asuteka Nagao for capreomycin and spectinomycin. ASKA library clones were obtained from the National Institute of Genetics, Japan. S.I. was supported by the Ministry of Education, Culture, Sports, Science and Technology (MEXT) [a Grant-in-Aid for Scientific Research on Innovative Areas “nascent chain biology" JP17H05679], the Japan Society for the Promotion of Science (JSPS) [a Grant-in-Aid for Young Scientists (A) JP17H04998, and a Challenging Research (Exploratory) JP19K22406], RIKEN (Pioneering Projects "Cellular Evolution" and Ageing Project), and the Takeda Science Foundation. H.T. was supported by MEXT (Grants-in-Aid for Scientific Research on Innovative Areas "nascent chain biology" JP26116002). T.I. was supported by MEXT (Grants-in-Aid for Scientific Research on Innovative Areas "nascent chain biology" JP15H01548 and JP17H05677), JSPS (Grant-in-Aid for Scientific Research [B] JP16H04756), RIKEN (Pioneering Project "Dynamic Structural Biology” and Ageing Project), and the Takeda Science Foundation. T.F. was supported by Grant-in-Aid for JSPS Fellows JP19J14480 from JSPS. This research was supported by Platform Project for Supporting Drug Discovery and Life Science Research [Basis for Supporting Innovative Drug Discovery and Life Science Research (BINDS)] from Japan Agency for Medical Research and Development (AMED) under Grant Number JP20am0101082. DNA libraries were sequenced by the Vincent J. Coates Genomics Sequencing Laboratory at UC Berkeley, supported by NIH S10 OD018174 Instrumentation Grant. 
Computations were supported by Manabu Ishii, Itoshi Nikaido, and the Bioinformatics Analysis Environment Service on RIKEN Cloud at RIKEN ACCC. T.F. was a recipient of the RIKEN Junior Research Associate Program and a JSPS Research Fellow (DC2). 


\section{$\underline{\text { Author Contributions }}$}

T.F. and S.I. performed the experiments; T.F. analyzed the deep sequencing data; T.Y. and T.I. performed the structural analysis; S.I. supervised the entire project with the help of H.T.; T.F. and S.I. wrote the manuscript; and all authors participated in editing the manuscript. 


\section{Experimental procedures}

\section{E. coli strains and DNA constructs}

The E. coli strains and plasmids used in this study are listed in Supplementary Table 2 and Supplementary Table 3. The ASKA library clones (Kitagawa et al., 2005) and Keio collection strains (Baba et al., 2006) were kind gifts from the National Institute of Genetics (NIG), Japan.

P1 bacteriophage-mediated transduction was conducted to introduce the $y c b Z$ deletion mutation in the Keio collection strain JW0938-KC (Baba et al., 2006) into MG1655, and then the FRT-Km ${ }^{\mathrm{R}}$-FRT cassette was removed from the chromosome of the $y c b Z$ deletion strain using $p C P 20$ (Datsenko and Wanner, 2000).

pCA24N-hybA, hybB, ispH, napD, nuoA, plsX, sse A, ykgF, and ycbZ

DNA fragments encoding the full-length ORFs were PCR-amplified from the MG1655 genome and inserted into $p C N 24 A$ (Kitagawa et al., 2005) by In-Fusion HD (TaKaRa).

pCA24N-ycbZmotif WT, I557A, E559A, R560A, I561A, W579A, L580A, W579AL580A, E559A-R560A-I561A, I557A-E559A-R560A-I561A-W579A-L580A, I584AP585A-N586A, P585A-N586A, and N586A

A DNA fragment encoding 30 amino acids from the stop codon ( $y c b Z$ motif WT) was PCR-amplified from the MG1655 genome and inserted into $p C A 24 N$ (Kitagawa et al., 2005). The mutations were introduced by site-directed mutagenesis. 
pCA24N-6xHis-HRV3C-ftsQ85-HA-ycbZmotif

Construct design was performed as described in earlier reports (Bischoff et al., 2014). DNA fragments encoding 85 amino acids of $f$ ts $Q$, the HRV 3C cleavage site, and the HAtag were inserted into the $p C A 24 N-y c b Z$ motif, to fuse in-frame with the ycbZ motif, by In-Fusion HD (TaKaRa). Tandem stop codons were further introduced downstream of the stop codon of the ycbZ motif.

\section{pCI-neo-1xEGFP-Fluc-EGFP and pCI-neo-2xEGFP-Fluc-EGFP}

DNA fragments encoding enhanced green fluoresce protein (EGFP) were PCR-amplified from $p C I-n e o-F l u c-E G F P$ (Gupta et al., 2011) and inserted into $p C I-n e o-F l u c-E G F P$ by In-Fusion HD (TaKaRa). The inserted EGFP was placed upstream of Fluc to fuse inframe. For $p C I-n e o-2 x E G F P-F l u c-E G F P$, two tandem EGFPs fragments were inserted.

\section{Ribosome profiling}

Cell sampling and library preparation were performed as previously described (McGlincy and Ingolia, 2017) with some modifications. A saturated culture of E. coli MG1655 cells, which were grown in LB media overnight at $37^{\circ} \mathrm{C}$, was diluted at a 1:100 ratio into fresh $\mathrm{LB}$ media and grown at $37^{\circ} \mathrm{C}$ to an $\mathrm{OD}_{600}$ of $0.4-0.5$. Cells were collected by filtration and flash-frozen in liquid nitrogen. The ice grains of the cell pellet and lysis buffer (20 $\mathrm{mM}$ Tris- $\mathrm{HCl} \mathrm{pH}$ 7.5, $150 \mathrm{mM} \mathrm{NH}_{4} \mathrm{Cl}, 10 \mathrm{mM} \mathrm{MgCl}_{2}, 5 \mathrm{mM} \mathrm{CaCl}_{2}, 1 \mathrm{mM}$ DTT, and 1\% Triton X-100) with translation inhibitors $(100 \mu \mathrm{g} / \mathrm{ml}$ chloramphenicol, $200 \mu \mathrm{g} / \mathrm{ml}$ linezolid, $200 \mu \mathrm{g} / \mathrm{ml}$ blasticidin, 200 or $2000 \mu \mathrm{g} / \mathrm{ml}$ capreomycin, 200 or $2000 \mu \mathrm{g} / \mathrm{ml}$ 
viomycin, 200 or $2000 \mu \mathrm{g} / \mathrm{ml}$ spectinomycin) were pulverized using a Multi-beads Shocker [Yasui Kikai, MB2200(S)]. After thawing at $4^{\circ} \mathrm{C}$, the lysate was treated with Turbo DNase (Thermo Fisher Scientific) at $25 \mathrm{U} / \mu 1$ for $10 \mathrm{~min}$ on ice and then clarified by centrifugation at $20,000 \times \mathrm{g}$ and $4^{\circ} \mathrm{C}$ for $10 \mathrm{~min}$. RNA concentration in lysate was measured by a Qubit RNA BR Assay Kit (Thermo Fisher Scientific). Thirty-five micrograms of RNA per sample was treated with $150 \mathrm{U}$ of Nuclease S7 Micrococcal nuclease from Staphylococcus aureus (Roche) for $45 \mathrm{~min}$ at $25^{\circ} \mathrm{C}$. RNA fragments ranging from 17 to $50 \mathrm{nt}$ were gel-excised as ribosome footprints. Library preparation was performed as previously described (McGlincy and Ingolia, 2017). A Ribo-Zero rRNA Removal Kit (Bacteria) (Illumina) was used to deplete rRNA contamination. The libraries were sequenced on a HiSeq4000 (Illumina).

After depleting the reads originating from noncoding RNAs, the remaining reads were mapped to the E. coli genome sequence (NC_000913.2). Empirically, we defined an A-site position, which is essentially the same as the $3^{\prime}$ assignment as described previously (Woolstenhulme et al., 2015). Polarity scores were calculated as previously described (Schuller et al., 2017).

Disome profiling data (GSE51052) (Subramaniam et al., 2014) were processed as in regular ribosome profiling data analysis.

\section{tRNA-seq}

tRNAs from in vitro translation reactions and RNCs (prepared as described below) were purified using a mirVanaTM miRNA Isolation Kit (Thermo Fisher Scientific). After the deacylation reaction, RNAs were dephosphorylated, linker-ligated, circularized, and 
PCR-amplified, following the ribosome profiling library preparation protocol. The libraries were sequenced on a HiSeq4000 (Illumina). Sequenced reads were mapped on the tRNA sequences provided by the genomic tRNA database (http://lowelab.ucsc.edu/GtRNAdb/Esch_coli_K12/).

\section{S1 irCLIP-seq}

The irCLIP linker was prepared as previously described (Zarnegar et al., 2016) with modifications.

DNA

oligonucleotides

$5^{\prime}-$ /5Phos/NNNNNJJJJJAGATCGGAAGAGCACACGTCTGAAAAA/N(6)Azide/-3'

were synthesized by GeneDesign. The nonstandard characters in the oligonucleotides represent the following: /5Phos/: $5^{\prime}$ monophosphate, $\mathrm{N}$ : random nucleotide, J: sample barcode sequence, and /N(6)Azide/: Azide-PEG4-NHS-Ester conjugated with amino C6 linker. In this study, the following sample barcode sequences were used: ATCGT, AGCTA, CGTAA, and CTAGA. After pre-adenylation of the DNA linkers by Mth ligase [New England Biolabs (NEB)] (McGlincy and Ingolia, 2017), IRDye 800CW DBCO (LICOR) was conjugated in PBS by CLICK reaction. The DNA linkers were further purified by an Oligo Clean \& Concentrator Kit (Zymo Research) and used for ligation reactions.

E. coli cells were collected and lysed as described in the "Ribosome profiling" section. The lysis buffer was supplemented with translation inhibitor $(100 \mu \mathrm{g} / \mathrm{ml}$ chloramphenicol or $2000 \mu \mathrm{g} / \mathrm{ml}$ capreomycin). One hundred microlitres of lysate containing $20 \mu \mathrm{g}$ of total RNA was placed on a Terasaki plate and exposed to $254 \mathrm{~nm} \mathrm{UV}$ light at $400 \mathrm{~mJ} / \mathrm{cm}^{2}$ on ice with a CL-1000 Ultraviolet Crosslinker (UVP). The sample was layered on $900 \mu \mathrm{l}$ of sucrose cushion buffer $(20 \mathrm{mM}$ Tris-HCl pH 7.5, $150 \mathrm{mM}$ 
$\mathrm{NH}_{4} \mathrm{Cl}, 10 \mathrm{mM} \mathrm{MgCl} 2,1 \mathrm{mM}$ DTT, and $1 \mathrm{M}$ sucrose) with $100 \mu \mathrm{g} / \mathrm{ml}$ chloramphenicol or $2000 \mu \mathrm{g} / \mathrm{ml}$ capreomycin and ultra-centrifuged at 100,000 rpm for $1 \mathrm{~h}$ with a TLA110 rotor (Beckman-Coulter) at $4^{\circ} \mathrm{C}$. The ribosome pellet was resuspended in $50 \mu 1$ of lysis buffer. To purify S1 protein on ribosomes, an anti-S1 antibody was immobilized on $15 \mu 1$ of Dynabeads Protein G (Thermo Fisher Scientific) equilibrated with cleavage buffer (20 $\mathrm{mM}$ Tris- $\mathrm{HCl} \mathrm{pH} 7.5,150 \mathrm{mM} \mathrm{NH}_{4} \mathrm{Cl}, 10 \mathrm{mM} \mathrm{MgCl}_{2}, 5 \mathrm{mM} \mathrm{CaCl}_{2}$, and $1 \mathrm{mM} \mathrm{DTT}$ ). Then, the beads were incubated with the resuspended ribosome solution for $1 \mathrm{~h}$ at $4^{\circ} \mathrm{C}$ and washed 5 times with $20 \mathrm{mM}$ Tris- $\mathrm{HCl} \mathrm{pH} 7.5,1 \mathrm{M} \mathrm{NH}_{4} \mathrm{Cl}, 2 \mathrm{mM}$ EDTA, $5 \mathrm{mM}$ $\mathrm{CaCl}_{2}, 1 \%$ Triton $\mathrm{X}-100,0.5 \% \mathrm{SDS}, 0.5 \%$ deoxycholate, and $1 \mathrm{mM}$ DTT, then twice with cleavage buffer. The on-bead RNase reaction was conducted with $0.01 \mathrm{U} / \mu 1 \mathrm{MNase}$ (TaKaRa) in $100 \mu 1$ of cleavage buffer at $25^{\circ} \mathrm{C}$ for $45 \mathrm{~min}$ in a Thermomixer (Eppendorf) with $15 \mathrm{~s}$ at $1400 \mathrm{rpm}$ and $90 \mathrm{~s}$ at rest. The beads were washed 3 times with reaction buffer containing $5 \mathrm{mM}$ EGTA instead of $\mathrm{CaCl}_{2}$. Immuno-purified RNAs on the beads were dephosphorylated with $10 \mathrm{U}$ of T4 PNK (NEB) in $10 \mu \mathrm{l}$ of $1 \times$ T4 Polynucleotide Kinase Reaction Buffer (NEB) at $37^{\circ} \mathrm{C}$ for $1 \mathrm{~h}$, then linker-ligated by the addition of 20 $\mu 1$ of reaction solution containing 3.3 U/ $\mu 1$ T4 RNA Ligase 2, truncated KQ (NEB), 1× T4 RNA Ligase Reaction Buffer (NEB), 17\% PEG8000 (NEB), and $0.25 \mu \mathrm{M}$ IR800labelled linker at $16^{\circ} \mathrm{C}$ overnight in a Thermomixer with $15 \mathrm{~s}$ at $1400 \mathrm{rpm}$ and $90 \mathrm{~s}$ at rest. The beads were washed 3 times with cleavage buffer. The precipitants were eluted by $1 \times$ Bolt LDS sample buffer (Thermo Fisher Scientific), run on Bolt 4-12\% Bis-Tris Plus Gel (Thermo Fisher Scientific) with Bolt MOPS SDS Running Buffer (Thermo Fisher Scientific), and transferred to nitrocellulose membrane with Trans-Blot Turbo (Bio-Rad). The infrared signal was detected by an Odyssey CLx Infrared Imaging System (LI-COR). The target region of the membrane containing RNAs was excised and treated with $0.2 \mathrm{mg}$ 
of Proteinase K (Roche) in $200 \mu \mathrm{l}$ of $100 \mathrm{mM}$ Tris-HCl pH 7.5, $50 \mathrm{mM} \mathrm{NaCl}, 1 \mathrm{mM}$ EDTA, and $0.2 \% \mathrm{SDS}$ at $50^{\circ} \mathrm{C}$ for $1 \mathrm{~h}$ in a Thermomixer with $15 \mathrm{~s}$ at $1000 \mathrm{rpm}$ and $30 \mathrm{~s}$ at rest. The RNAs were purified by a Direct-zol MicroPrep Kit (Zymo Research). The downstream procedure for library preparation was followed by a ribosome profiling protocol (McGlincy and Ingolia, 2017). rRNA contamination was depleted by the RiboZero rRNA Removal Kit (Bacteria) (Illumina). The libraries were sequenced by HiSeq4000 (Illumina).

Reads were analysed as described in the "Ribosome profiling" section. Reads at a given position were normalized by average reads per nucleotide at each gene.

\section{DMS-seq data analysis}

DMS-seq data from E. coli K-12 MG1655 (GSE77617) (Burkhardt et al., 2017) were processed as described in the "Ribosome profiling" section. The score at a given position was the average of reads in a 33 -nucleotide window. Data obtained at $37^{\circ} \mathrm{C}$ were compared to those at $95^{\circ} \mathrm{C}$.

\section{Cryo-EM single particle analysis}

\section{Sample preparation}

E. coli cells were collected and lysed as described in the "Ribosome profiling" section.

Cell lysate was layered on top of a 10-50\% sucrose density gradient containing $20 \mathrm{mM}$ Tris- $\mathrm{HCl} \mathrm{pH}$ 7.5, $150 \mathrm{mM} \mathrm{NH} 4 \mathrm{Cl}, 10 \mathrm{mM} \mathrm{MgCl}_{2}, 1 \mathrm{mM}$ DTT, $100 \mu \mathrm{g} / \mathrm{ml}$ chloramphenicol or $200 \mu \mathrm{g} / \mathrm{ml}$ capreomycin, and $2 \mathrm{U} / \mathrm{ml}$ SUPERase $\bullet$ In RNase inhibitor 
(Thermo Fisher Scientific) and centrifuged at 35,300 rpm and $4^{\circ} \mathrm{C}$ for $2.5 \mathrm{~h}$ with P40ST Rotor (HITACHI). Samples were fractionated using Gradient Station (Biocomp) with MICRO COLLECTOR AC-5700 (ATTO). The absorbance was detected by BIO MINI UV MONITOR AC-5200S (ATTO). Polysome fractions were pooled, buffer-exchanged to grid buffer (200 mM Tris- $\mathrm{HCl} \mathrm{pH} 7.5,150 \mathrm{mM} \mathrm{NH} 4 \mathrm{Cl}, 10 \mathrm{mM} \mathrm{MgCl}_{2}$, and $1 \mathrm{mM}$ DTT) with translation inhibitor (100 $\mu \mathrm{g} / \mathrm{ml}$ chloramphenicol or $2000 \mu \mathrm{g} / \mathrm{ml}$ capreomycin) with Amicon Ultra-15 Centrifugal Filters 100K (Millipore), flash-frozen by liquid nitrogen, and stored at $-80^{\circ} \mathrm{C}$.

For the RNC, in vitro transcription and translation were performed with PURE flex 1.0 (GeneFrontier) at $37^{\circ} \mathrm{C}$ for $30 \mathrm{~min}$ following the manufacturer's instructions. The DNA fragment was amplified from $p C A 24 N-6 x H i s-H R V 3 C-f t s Q 85-H A-y c b Z m o t i f$ with primer 1 GGCCTAATACGACTCACTATAGGAGAAATCATAAAAAATTTATTTGCTTTGT GAGCGG-3') and primer3 (5'-AGTCAGTCACGATGAATTCCCCTAGCTTGG-3') (Chadani et al., 2016) and used for the assay. The reaction was layered on $900 \mu 1$ of modified sucrose cushion buffer $[20 \mathrm{mM}$ Tris- $\mathrm{HCl} \mathrm{pH} 6.8,150 \mathrm{mM} \mathrm{NH} 4 \mathrm{Cl}, 50 \mathrm{mM}$ $\mathrm{MgCl}_{2}, 5 \mathrm{mM}$ 2-mercaptoethanol, $0.1 \%$ (v/v) Nikkol, and $1 \mathrm{M}$ sucrose] and ultracentrifuged at 100,000 rpm for $1 \mathrm{~h}$ with a TLA110 rotor (Beckman-Coulter) at $4^{\circ} \mathrm{C}$. The pellet was resuspended in resuspension buffer $\left[20 \mathrm{mM}\right.$ Tris- $\mathrm{HCl} \mathrm{pH} 6.8,150 \mathrm{mM} \mathrm{NH}_{4} \mathrm{Cl}$, $50 \mathrm{mM} \mathrm{MgCl} 2,5 \mathrm{mM}$ 2-mercaptoethanol, and $0.1 \%$ (v/v) Nikkol]. To affinity-purify the RNC, $1200 \mu \mathrm{l}$ of TALON Metal Affinity Resin (Clontech) was equilibrated with resuspension buffer and transferred to Mini Bio-Spin Chromatography Columns (BioRad). The resuspended RNC was incubated with the resin within the column for $20 \mathrm{~min}$ at $4^{\circ} \mathrm{C}$. The column was centrifuged at $700 \times \mathrm{g}$ for 1 min and washed with $5 \mathrm{ml}$ 
resuspension buffer in total. The RNC complexes trapped on beads were eluted with 2.5 $\mathrm{ml}$ of elution buffer [20 mM Tris- $\mathrm{HCl} \mathrm{pH} 6.8,150 \mathrm{mM} \mathrm{NH}_{4} \mathrm{Cl}, 50 \mathrm{mM} \mathrm{MgCl}, 5 \mathrm{mM} 2-$ mercaptoethanol, $0.1 \%(\mathrm{v} / \mathrm{v}) \mathrm{Nikkol}$, and $250 \mathrm{mM}$ imidazole $\mathrm{pH}$ 7.0] and further purified by sucrose cushion as mentioned above. The pellet was resuspended in resuspension buffer containing $10 \mathrm{mM}$ DTT instead of $5 \mathrm{mM}$ 2-mercaptoethanol. The solution was layered on top of a 10-50\% sucrose density gradient containing $20 \mathrm{mM}$ Tris- $\mathrm{HCl} \mathrm{pH} 6.8$, $150 \mathrm{mM} \mathrm{NH}_{4} \mathrm{Cl}, 50 \mathrm{mM} \mathrm{MgCl}_{2}, 10 \mathrm{mM}$ DTT, and $0.1 \%$ (v/v) Nikkol and centrifuged at $14,400 \mathrm{rpm}$ and $4^{\circ} \mathrm{C}$ for $15 \mathrm{~h}$ with a P40ST Rotor (HITACHI). The $70 \mathrm{~S}$ fraction was pooled and concentrated by sucrose cushion as described above. Finally, the pellet was resuspended in grid buffer [20 mM Tris- $\mathrm{HCl} \mathrm{pH} 6.8,150 \mathrm{mM} \mathrm{NH} 4 \mathrm{Cl}, 50 \mathrm{mM} \mathrm{MgCl}_{2}, 2$ mM DTT, and $0.1 \%(\mathrm{v} / \mathrm{v}) \mathrm{Nikkol}$, flash-frozen by liquid nitrogen, and stored at $-80^{\circ} \mathrm{C}$.

\section{Cryo-EM grid preparation, image acquisition, and data processing}

For cryo-EM grid preparation, concentrations of three ribosome specimens including 70S with chloramphenicol, 70S with capreomycin, and ycbZ-stalled $70 \mathrm{~S}$ were adjusted to 50 nM. A $3 \mu \mathrm{L}$ volumes of ribosome specimen was applied onto Quantifoil R1.2/1.3 300 mesh $\mathrm{Cu}$ grids (Quantifoil) coated with a homemade thin amorphous carbon layer. Grids were glow discharged using a PID-10 Plasma Ion Bombarder (Vacuum Device) at $5 \mathrm{~mA}$ for $10 \mathrm{~s}$ before use. An excess amount of solution was blotted off with filter papers and immediately plunged into liquid ethane for vitrification using Vitrobot Mark IV (Thermo Fisher Scientific). Ribosome specimens were imaged using a Tecnai Arctica transmission electron microscope (Thermo Fisher Scientific) operated at $200 \mathrm{kV}$ accelerating voltage and equipped with a K2 summit direct electron detector (Gatan). Automated image acquisition was performed with Serial EM program (Mastronarde, 2005) at the nominal 
magnification of $x 23,500$, which is corresponding an objective pixel size at the specimen level of $1.47 \AA$. It should be noted that the data collection of specimens with antibiotics were performed on super-resolution mode. Therefore, pixel size became two times finer, which is corresponding to $0.735 \AA$ shown in Table S1. The total exposure of electrons at the specimen level was around 50e- $\AA-2$ and dose fractionated into 40 frames (Table S1). Images were recoded as movie micrographs. Image processing were performed using RELION3 (Zivanov et al., 2018). Details of image processing are described as follows.

1) The dataset of the chloramphenicol-bound E. coli 70S ribosome

Obtained movie micrographs in super-resolution mode were motion corrected using the program implemented in RELION with applying $2 \mathrm{x}$ binning by fourier cropping. CTF parameters were estimated by CTFFIND4.1 program (Rohou and Grigorieff, 2015). Ribosome particles were automatically picked by Gautomatch program (https://www.mrc-lmb.cam.ac.uk/kzhang/Gautomatch/).

Obtained $165 \mathrm{~K}$ particles were initially subjected to $2 \mathrm{D}$ classification to discard 30S and junk particles (Supplementary Fig. 2a). 157K remained particles were autorefined to reconstruct a consensus map. Subsequently, based on the alignment of consensus reconstruction, 3D classification was performed (Supplementary Fig. 2a). As a result of 3D classification, the data set was classified into three subgroups: $70 \mathrm{~S}$ in the rotated state $(35 \%)$, dissociated $50 \mathrm{~S}(5 \%)$, and $70 \mathrm{~S}$ in the non-rotated state $(60 \%)$. Rotated (55K particles) and nonrotated (94K particles) $70 \mathrm{~S}$ subgroups were further auto-refined. In addition, CTF-refinement, Bayesian polishing, and another round of auto-refinement were performed. Eventually, 70S ribosomes with chloramphenicol bindings in the rotated or nonrotated states both at resolution of $3.0 \AA$ were obtained (Supplementary Fig. 2a, c). 
Local resolution distribution on obtained cryo-EM maps were calculated using the program implemented in RELION (Supplementary Fig. 2e, f).

2) The dataset of the capreomycin-bound E. coli 70 S ribosome

Movie micrographs recorded on super-resolution mode were motion-corrected using the program implemented in RELION by $2 \mathrm{x}$ binning as described above. CTF parameters were estimated by CTFFIND4.1 program (Rohou and Grigorieff, 2015). Automated particle picking was performed with Gautomatch program. Obtained 158K particles were 2D classified to discard 30S and junk particles (Supplementary Fig. 2b). Remained 154K particles converged to a consensus reconstruction by auto-refinement. Using this alignment information, the dataset was subject to 3D classification (Supplementary Fig. 2b). The dataset was sorted into three subgroups: dissociated 50S (3\%), 70S in the nonrotated state $(41 \%)$, and $70 \mathrm{~S}$ in the rotated state (52\%). A subgroup of the rotated $70 \mathrm{~S}$ (81K particles) was further auto-refined. Subsequently, CTF-refinement, Bayesian polishing, and auto-refinement was performed. Final reconstructed structure was at the resolution of $3.0 \AA$ (Supplementary Fig. 2b, c). Local resolution was estimated using the program implemented in RELION (Supplementary Fig. 2g).

3) The dataset of the ycbZ-stalled E. coli $70 \mathrm{~S}$ ribosome

Movie micrographs were performed using the program implemented in RELION. CTF parameters were estimated by CTFFIND4.1 program (Rohou and Grigorieff, 2015). Particle picking was performed with Gautomatch program. Obtained $77 \mathrm{~K}$ particles was subject to 2D classification to discard $30 \mathrm{~S}$ and junk particles. Remained $63 \mathrm{~K}$ particles were auto-refined to a consensus reconstruction. The dataset subject to 3D classification. 
Particles of 70S in the non-rotated state represented the major population (Supplementary Fig. 6c). This data set was further auto-refined and subsequently CTF refinement, Bayesian polishing, and another auto-refinement were performed. The obtained final reconstruction was at the resolution of $3.3 \AA$ (Supplementary Fig. 2c, 6c). Local resolution distribution was calculated using the program implemented in RELION (Supplementary Fig. 2h).

\section{Model building and refinement}

Initial model building was performed using UCSF chimera (Pettersen et al., 2004) and COOT (Emsley et al., 2010). Briefly, 70S portion of PDB: 5AFI (Fischer et al., 2015) was ridged body fitted to obtained cryo-EM maps. Subsequently, tRNA (PDB: 5AFI) in antibiotics bonding 70S and tRNAPro (PDB: 6ENJ) (Huter et al., 2017) in ycbZ-stalled 70S were ridged body fitted. Theses initial models were real-space refined by phenix.real_space_refine routine to cryo-EM maps (Adams et al., 2010). Refined final models were validated using MOLPROBITY (Chen et al., 2010). Map vs. model FSC were calculated in Phenix (Supplementary Fig. 2d) (Adams et al., 2010). Graphical figures were prepared by UCSF Chimera (Pettersen et al., 2004) and UCSF Chimera X (Goddard et al., 2018).

\section{Integrated nascent chain profiling (iNP)}

iNP was performed as previously described (Chadani et al., 2016). The sample was run on Bolt 12\% Bis-Tris Plus gel (Thermo Fisher Scientific) with Bolt MES SDS Running Buffer (Thermo Fisher Scientific). 


\section{Western blot analysis}

Anti-S1 (a kind gift from the Christian Spahn laboratory, 1:1000) (Duval et al., 2013), anti-S2 (Abcam, ab193293, 1:1000), and anti-HA-tag (MBL science, M180-3, 1:1000) were used as primary antibodies. Infrared fluorescence Western blotting was conducted as previously described (Iwasaki et al., 2019).

\section{Preparation of the S1-depleted $70 S$ ribosomes}

S1 was biochemically depleted from ribosomes as previously described (Duval et al., 2013) with some modifications. PolyU Sepharose 4B (GE Healthcare) and Pierce Control Agarose Resin (Thermo Fisher Scientific) (as a control) were equilibrated with equilibration buffer [20 mM Tris- $\mathrm{HCl} \mathrm{pH} 7.5,150 \mathrm{mM} \mathrm{NH}_{4} \mathrm{Cl}, 50 \mathrm{mM} \mathrm{MgCl}_{2}$, and $1 \mathrm{mM}$ DTT] and transferred to Mini Bio-Spin Chromatography Columns (Bio-Rad). The resuspended ribosome solution $(1 \mu \mathrm{M})$ (PURE flex1.0, GeneFrontier) was incubated with the resin within the column for $1 \mathrm{~h}$ at $4^{\circ} \mathrm{C}$. The column was centrifuged at $700 \times \mathrm{g}$ for 1 min and the flow-through fraction was collected.

\section{Luciferase reporter assay with in vitro translation system}

DNA fragments were amplified from $p C I-n e o-F l u c-E G F P$ with forwad primer 5'TAATACGACTCACTATAGGatacgcacaataaggctattgtacgtatgcaaattaataataaaggagagtagc aatgtcattcgaattacctgcaatggaagacgccaaaaaca $\quad-3^{\prime}$ and reverse primer 5'- 
GTTATTGCTCAGCggttagcggecgcataggcggtggatccegggttt -3'. For pCI-neo-1xEGFPFluc-EGFP and pCI-neo-2xEGFP-Fluc-EGFP, a oligonucleotide 5'TAATACGACTCACTATAGGatacgcacaataaggctattgtacgtatgcaaattaataataaaggagagtagc aatgtcattcgaattacctgcaGGGTCGACCTGCAGC $-3^{\prime}$ was used as forward primer. Using these DNA fragments as templates, mRNAs were prepared by T7-Scribe Standard RNA IVT Kit (CELLSCRIPT).

In vitro translation was conducted with PURE flex 1.0 (GeneFrontier), using S1-depleted or mock-treated ribosomes, at $37^{\circ} \mathrm{C}$ for $30 \mathrm{~min}$. Luminescence was detected with a dual-luciferase reporter assay system (Promega) and GloMax (Promega). 


\section{Figure and Table Legends}

Fig. 1: Differential sizes of ribosome-protected fragments generated by translation inhibitors.

(a and b) Footprint size distributions in ribosome profiling with peptidyl transfer inhibitors (a) and translocation inhibitors (b).

(c and d). Normalized ribosome occupancy of the indicated size of footprints with chloramphenicol (c) and capreomycin (d) treatments around the start codon.

(e and f) Ribosome conformation revealed by cryo-EM under chloramphenicol (e) and capreomycin (f) treatments.

(g) A close-up view of chloramphenicol binding to PTC shown with the ribosomal environment. 50S subunit is removed to show the details.

(h) A close-up view of capreomycin binding to the $16 \mathrm{~S}$ decoding center shown with the ribosomal environment. 50S subunit is removed to show the details.

( $\mathrm{i}$ and $\mathrm{j}$ ) The footprint distribution at the A-site position of reads along tnaC (i) and $\sec M$

(j). The indicated sizes of footprints are depicted. The known pause sites are indicated by arrows.

RPM: reads per million mapped reads

Fig. 2: S1 protein extends footprints in the rotated state of ribosomes.

(a) Meta-gene analysis showing the $5^{\prime}$ and $3^{\prime}$ ends of footprints around stop codons. Short (23-28 nt) (top) and long (35-40 nt) (bottom) chloramphenicol- and capreomycin-treated samples, respectively, are shown. 
(b and c) Footprint size distributions from S1 WT, S1 $\Delta 6$, and S1 $\Delta 56$ strains with chloramphenicol (b) and with capreomycin (c).

(d) Read enrichment of S1 irCLIP with capreomycin (cap) over chloramphenicol (cm) treatment, around stop codon. The $5^{\prime}$ end of reads is shown. The enriched site, which is corresponded to the footprint extension at the stop codon, is indicated by black arrow.

\section{Fig. 3: S1 facilitates translation elongation from long CDS.}

(a) The difference of polarity scores of footprints in S1 $\Delta 56$ strain compared S1 WT strain. The mRNAs were sub-classed into four groups according to the CDS length.

(b) Schematic representation of reporters constructed (top). sodB 5' UTR, which enables S1-independent translation initiation, is fused to firefly luciferase (Fluc). Single or tandem GFPs were inserted to extend CDS length. These reporters were translated by S1depleted ribosomes with in vitro translation system (bottom). Data represent mean and S.D. $(n=3)$.

Fig. 4: $y c b Z$ mRNA has a ribosome pause site with long footprints.

(a) Scatterplot representing long footprint (35-40 nt) versus short footprint (23-27 nt) accumulation across all codons of the transcriptome without drug treatment. Light purple: codons with long footprint over-represented, which are defined as long footprint pause scores larger than mean +11.5 S.D. and a ratio of long footprint to short footprint larger than 0.8; blue: sites tested for iNP in (c), and dark purple: known arrest sites in secM and tna $\mathrm{C}$ mRNAs. \# and following number stand for A-site position of codon. 
(b) Scatterplot representing the ratio of long footprint (35-40 nt) to short footprint (23-27

nt) at the selected codon positions in drug-free and chloramphenicol-treated conditions.

(c) iNP for selected mRNAs highlighted in (a) and (b). RNase-sensitive peptidyl-tRNAs are indicated by arrowheads.

Fig. 5: Ribosomes stalled at the $y c b Z$ pause site revealed by cryo-EM.

(a) The footprint distribution at the A-site position of reads along $y c b Z$. The indicated sizes of footprints are depicted. The tested pause sites are indicated by an arrow.

(b) Same as (a) but for disome footprints reported in (Subramaniam et al., 2014).

(c) Schematic representation of reporter constructed (top) and Western blot for peptidyltRNA (probed by HA epitope) from the reporter run in SDS-PAGE under natural $\mathrm{pH}$ (bottom). RNase treatment verified the RNA conjugation.

(d) Enrichment of tRNAs in the RNC at the $y c b Z$ pause site, assessed by tRNA-seq. (e and f) Close-up view of the inter-subunit cavity of this complex depicting the structural details of the nascent peptide-bound P-site tRNA (e). The structural model of tRNA ${ }^{\text {Pro }}{ }_{\text {CGG }}$ is well fitted to the corresponding cryo-EM density. The details of the codon-anticodon recognition of P-site tRNA are shown in (f).

(g) RNA base-paring around the $y c b Z$ pause site (indicated by an arrow), probed by DMSseq (Burkhardt et al., 2017). Reads in a 33 nt window were summed and normalized. Red shade shows footprint extended region upstream of A site.

Fig. 6: Schematic representation of bacterial footprint extension.

Fujita et al. p36 
During bacterial ribosome profiling, short footprints (23-27 nt) are originated from nonrotated ribosomes, which could be trapped by chloramphenicol. Long footprints (35-40 nt) are generated by subunit rotation. Rotated ribosome (stabilized by capreomycin) leads to high probability of mRNA association by S1, which sterically inhibits a RNase attack. A minor fraction of long footprints are originated by intra-molecular and inter-molecular (SD/andi-SD) base-paring, that are resistant to RNase digestion.

\section{Supplementary Fig. 1: Ribosome footprint size altered by translation inhibitors.}

(a-c) Footprint size distribution with low and high concentrations of capreomycin (a), viomycin (b), and spectinomycin (c).

(d) Correlation of footprint reads on CDSs between chloramphenicol- and capreomycintreated samples.

(e) Correlation of ribosome occupancy on A-site codons calculated from short footprints and long footprints enriched by chloramphenicol and capreomycin treatment, respectively.

$r$ : Pearson's correlation, $\rho$ : Spearman's rank correlation.

\section{Supplementary Fig. 2: Characterizations of ribosome structures trapped by drugs.}

(a) Image processing of $70 \mathrm{~S}$ ribosomes with chloramphenicol addition. Briefly, $165 \mathrm{~K}$ particles were extracted from motion-corrected movie-micrographs followed by 2D classification to discard bad particles. Remained $157 \mathrm{~K}$ particles were auto-refined to obtain the consensus reconstruction. Based on the alignment information of this 
reconstruction, 3D classification with local angular search was performed. Classification was converged into three structurally homogeneous subgroups; 50S (5\%), a rotated $70 \mathrm{~S}$ $(35 \%)$, and a non-rotated $70 \mathrm{~S}(60 \%)$. Rotated and non-rotated $70 \mathrm{Ss}$ were further processed through auto-refinement, CTF refinement, bayesian polishing, and autorefinement. Obtained cryo-EM structures reach $3.0 \AA$ in the rotated $70 \mathrm{~S}$ and $3.0 \AA$ in the nonrotated $70 \mathrm{~S}$.

(b) Image processing of 70S ribosomes with capreomycin addition, performed in the same way as (A). Briefly, the extracted $158 \mathrm{~K}$ particles were $2 \mathrm{D}$ classified to obtain good particles (154K particles). Remained particles were converged into the consensus reconstruction. Subsequently, 3D classification was performed and converged into three subgroups, 50S (3\%), a rotated 70S (52\%), and a non-rotated 70S (41\%). Since the nonrotated ribosomes were tRNA-free, we focused on the rotated ribosomes. The rotated 70S with two tRNA binding were further processed as the same way described above. The obtained 70S ribosome in a rotated state is at the resolution of $3.0 \AA$.

(c and d) Fourier shell correlation curves of four cryo-EM structures (c) and models vs. cryo-EM maps (d) are shown.

(e-h) Color-coded local resolution distributions of cryo-EM maps for chloramphenicolbound $70 \mathrm{~S}$ in a non-rotated state (e), chloramphenicol-bound $70 \mathrm{~S}$ in a rotated state (f), capreomycin-bound $70 \mathrm{~S}$ in a rotated state $(\mathrm{g})$, and $y c b Z$ stalled $70 \mathrm{~S}(\mathrm{~h})$.

(i) Structural comparison of 70S ribosomes with chloramphenicol bindings in nonrotated and rotated states. Two structures are aligned using $50 \mathrm{~S}$ subunits as guides and only $30 \mathrm{~S}$ subunits were shown. 
(j) Structural comparison of the $70 \mathrm{~S}$ ribosome with capreomycin binding in a rotated state and the $70 \mathrm{~S}$ ribosome with chloramphenicol binding in a rotated state. $30 \mathrm{~S}$ densities are nearly matched each other.

\section{Supplementary Fig. 3: Characterization of S1 irCLIP-seq.}

(a) Schematic representation of mutant strains of S1 (Duval et al., 2013). The endogenous

$\mathrm{S} 1$ gene was complemented by the S1 WT, S1 $\Delta 6$, or S1 $\Delta 56$ gene with kan caseate.

(b) Western blot for S1 protein in the mutant strains.

(c) Immunopurified S1 protein (left) and super-shift by the conjugation of crosslinked RNA ligated to IR800-labeled linker (right). HC: antibody heavy chain, LC: antibody light chain, CBB: Coomassie Brilliant Blue.

(d) Meta-gene analysis of S1 irCLIP-seq reads around the start codon with chloramphenicol (top)- and capreomycin (bottom)-treated samples. The 5' end of the reads is depicted.

Supplementary Fig. 4: Characterization of mRNAs with codons where long footprints are over-represented.

(a) Relative enrichment of short (23-27 nt) and long (35-40 nt) footprints around the UGG codon, which represents an anti-SD-like sequence.

(b) Footprint size distribution of the chloramphenicol-treated sample. The distribution was fitted to two populations by Gaussian distribution (dark blue), which are sum of distributions peaked at $\sim 25 \mathrm{nt}$ and $\sim 35 \mathrm{nt}$ (light blue).

Fujita et al. p39 
(c) $\triangle \mathrm{G}$ value between the anti-SD sequence (5'-ACCUCCUUA-3') and the sequence upstream (19 nt) of the pause site. Broken lines represent the average $\Delta \mathrm{G}$ value of all pairs of typical SD (19 nt upstream of start codons) and anti-SD sequences (red) and the range of one standard distribution (black).

(d) iNP for selected mRNAs highlighted in Fig. 4a, b.

Supplementary Fig. 5: Characterization of the $y c b Z$ pause site where long footprints are over-represented.

( $a$ and $b$ ) The zoomed-out view of ribosome profiling (a) and disome profiling (b) reads on $y c b Z$.

(c) Conservation of the $y c b Z$ pause motif (the amino acid position in E. coli is shown). Mutated amino acids are indicated by arrowheads.

(d) Western blot for peptidyl-tRNA (probed by HA epitope) from the reporters run in natural pH SDS-PAGE.

(e) Schematic representation of the RNC purification procedure.

(f) Western blot for peptidyl-tRNA probed by HA-tag (left) and CBB staining of proteins in RNC used for cryo-EM analysis (right).

(g) Sucrose density gradient of immunopurified RNCs. The 70S fraction was used for downstream cryo-EM analysis.

Supplementary Fig. 6: Purification of RNCs on the $y c b Z$ pause site.

Fujita et al. p40 
(a) RNA secondary structure prediction of $60 \mathrm{nt}$ from A-site at pause site with long footprint accumulation by CentroidFold (Sato et al., 2009). Footprint extended regions are shaded in red.

(b) RNA base-paring around pause sites (indicated by arrows) with long footprint accumulation, probed by DMS-seq (Burkhardt et al., 2017). Reads in a 33 nt window were summed and normalized. Red shades show footprint extended region upstream of A site.

(c) Image processing of $70 \mathrm{~S}$ ribosomes stalled with $y c b Z$. $77 \mathrm{~K}$ particles were extracted followed by $2 \mathrm{D}$ classification to obtain good particles (63K particles). The dataset was converged into the consensus reconstruction and 3D classification was performed. The dataset classified into six subgroups. The major population (64\%) assigned to $70 \mathrm{~S}$ ribosome in a non-rotated state was further processed. The obtained refined cryo-EM structure is at the resolution of $3.3 \AA$.

Supplementary Table 1: Cryo-EM data collection, model building, refinement, and validation statistics.

\begin{tabular}{|c|c|c|c|c|}
\hline & $\begin{array}{c}\text { Chrolamphenicol/ } \\
\text { Nonrotated) }\end{array}$ & $\begin{array}{c}\text { (Chrolamphenicol/ } \\
\text { Rotated) }\end{array}$ & $\begin{array}{l}\text { (Capreomycin/ } \\
\text { Rotated) }\end{array}$ & (ycbZ-stalled) \\
\hline Data collection and proce & & & & \\
\hline Magnification & \multicolumn{4}{|c|}{23,500} \\
\hline Voltage $(\mathrm{kV})$ & \multicolumn{4}{|c|}{200} \\
\hline Electron exposure $\left(\mathrm{e}^{-} / \AA^{2}\right)$ & \multicolumn{4}{|c|}{50} \\
\hline Defocus range $(\mu \mathrm{m})$ & \multicolumn{2}{|c|}{$0.5-3.0$} & $0.5-3.5$ & $1.0-3.0$ \\
\hline Pixel size $(\AA)$ & \multicolumn{2}{|c|}{0.735} & 0.735 & 1.47 \\
\hline Symmetry & & $C_{1}$ & & \\
\hline
\end{tabular}




\begin{tabular}{|c|c|c|c|c|}
\hline \multirow{2}{*}{$\begin{array}{l}\text { Initial particle images (no.) } \\
\text { Final particle images (no.) }\end{array}$} & \multicolumn{2}{|c|}{164,655} & \multirow{2}{*}{$\begin{array}{l}158,017 \\
80,606\end{array}$} & \multirow{2}{*}{$\begin{array}{r}77,002 \\
40,231\end{array}$} \\
\hline & 94,218 & 54,801 & & \\
\hline Map resolution $(\AA)$ & 3.0 & 3.0 & 3.0 & 3.3 \\
\hline FSC threshold & 0.143 & 0.143 & 0.143 & 0.143 \\
\hline Map sharpening $B$ factor $\left(\AA^{2}\right)$ & -33.52 & -31.28 & -34.78 & -64.57 \\
\hline \multicolumn{5}{|l|}{ Refinement } \\
\hline Model composition & & & & \\
\hline Non-hydrogen atoms & 146,057 & 146,057 & 146,277 & 145,941 \\
\hline Protein residues & 5,949 & 5,949 & 5,949 & 5,951 \\
\hline RNA residues & 4,645 & 4,645 & 4,654 & 4,639 \\
\hline \multicolumn{5}{|l|}{ R.m.s. deviations } \\
\hline Bond lengths $(\AA)$ & 0.005 & 0.006 & 0.009 & 0.010 \\
\hline Bond angles $\left(^{\circ}\right)$ & 0.663 & 0.701 & 0.815 & 0.884 \\
\hline \multicolumn{5}{|l|}{ Validation } \\
\hline MolProbity score & 2.26 & 2.33 & 2.34 & 2.26 \\
\hline Clashscore & 14.79 & 16.68 & 17.11 & 12.63 \\
\hline Poor rotamers $(\%)$ & 0.04 & 0.12 & 0.31 & 0.29 \\
\hline RNA pucker outliers (\%) & 0 & 0 & 0 & 0 \\
\hline \multicolumn{5}{|l|}{ Ramachandran plot } \\
\hline Favored (\%) & 88.83 & 87.84 & 87.55 & 85.70 \\
\hline Allowed (\%) & 10.74 & 11.73 & 11.94 & 13.82 \\
\hline Disallowed (\%) & 0.43 & 0.43 & 0.51 & 0.48 \\
\hline
\end{tabular}

Supplementary Table 2: A list of $E$. coli strains used in this study.

Strain

MG1655

MG1655 rpsA1 (MS71)
Reference

Laboratory stock

Duval et al., 2013 
MG1655 rpsA $\Delta 6$ (MS72)

MG1655 rpsA $\Delta 56$ (MS73)
Duval et al., 2013

Duval et al., 2013

\section{Supplementary Table 3: A list of plasmids used in this study.}

Plasmid

pCA24N-secM

pCA24N-hybA

$p C A 24 N-h y b B$

$p C A 24 N-i s p H$

pCA24N-napD

pCA24N-nиоA

pCA24N-plsX

pCA24N-sseA

$p C A 24 N-y k g F$

$p C A 24 N-y c b Z$

pCA24N-ycbZmotif

pCA24N-ycbZmotif (I557A)

pCA24N-ycbZmotif (E559A)

pCA24N-ycbZmotif (R560A)

pCA24N-ycbZmotif (I561A)

pCA24N-ycbZmotif (W579A)

pCA24N-ycbZmotif (L580A)
Reference

Kitagawa et al., 2005

This paper

This paper

This paper

This paper

This paper

This paper

This paper

This paper

This paper

This paper

This paper

This paper

This paper

This paper

This paper

This paper 


\begin{tabular}{|c|c|}
\hline$p C A 24 N-y c b Z m o t i f(W 579 A-L 580 A)$ & This paper \\
\hline$p C A 24 N-y c b Z m o t i f(E 559 A-R 560 A-I 561 A)$ & This paper \\
\hline $\begin{array}{l}\text { pCA24N-ycbZmotif } \quad(\text { I557A-E559A-R560A-I561A-W579A- } \\
\text { L580A) }\end{array}$ & This paper \\
\hline$p C A 24 N-y c b Z m o t i f(I 584 A-P 585 A-N 586 A)$ & This paper \\
\hline$p C A 24 N-y c b Z m o t i f(P 585 A-N 586 A)$ & This paper \\
\hline$p C A 24 N-y c b Z m o t i f(N 586 A)$ & This paper \\
\hline pCA24N-6xHis-HRV3C-ftsQ85-HA-ycbZmotif & This paper \\
\hline pCI-neo-Fluc-EGFP & Gupta et al., 2011 \\
\hline pCI-neo-1xEGFP-Fluc-EGFP & This paper \\
\hline pCI-neo-2xEGFP-Fluc-EGFP & This paper \\
\hline
\end{tabular}




\section{$\underline{\text { References }}$}

Adams, P. D., Afonine, P. V., Bunkóczi, G., Chen, V. B., Davis, I. W., Echols, N., Headd, J. J., Hung, L. W., Kapral, G. J., Grosse-Kunstleve, R. W., McCoy, A. J., Moriarty, N. W., Oeffner, R., Read, R. J., Richardson, D. C., Richardson, J. S., Terwilliger, T. C., and Zwart, P. H. (2010). PHENIX: a comprehensive Python-based system for macromolecular structure solution. Acta Crystallogr D Biol Crystallogr 66, $213-221$.

Baba, T., Ara, T., Hasegawa, M., Takai, Y., Okumura, Y., Baba, M., Datsenko, K. A., Tomita, M., Wanner, B. L., and Mori, H. (2006). Construction of Escherichia coli K-12 in-frame, single-gene knockout mutants: the Keio collection. Mol Syst Biol 2, 2006.0008 .

Beckert, B., Turk, M., Czech, A., Berninghausen, O., Beckmann, R., Ignatova, Z., Plitzko, J. M., and Wilson, D. N. (2018). Structure of a hibernating 100S ribosome reveals an inactive conformation of the ribosomal protein S1. Nat Microbiol 3, 11151121.

Bischoff, L., Berninghausen, O., and Beckmann, R. (2014). Molecular basis for the ribosome functioning as an L-tryptophan sensor. Cell Rep 9, 469-475.

Boni, I. V., Isaeva, D. M., Musychenko, M. L., and Tzareva, N. V. (1991). Ribosomemessenger recognition: mRNA target sites for ribosomal protein S1. Nucleic Acids Res $19,155-162$.

Borovinskaya, M. A., Shoji, S., Holton, J. M., Fredrick, K., and Cate, J. H. D. (2007). A steric block in translation caused by the antibiotic spectinomycin. ACS Chem Biol 2, $545-552$. 
Brandman, O., Stewart-Ornstein, J., Wong, D., Larson, A., Williams, C. C., Li, G. W., Zhou, S., King, D., Shen, P. S., Weibezahn, J., Dunn, J. G., Rouskin, S., Inada, T., Frost, A., and Weissman, J. S. (2012). A ribosome-bound quality control complex triggers degradation of nascent peptides and signals translation stress. Cell 151, 10421054.

Brar, G. A., and Weissman, J. S. (2015). Ribosome profiling reveals the what, when, where and how of protein synthesis. Nat Rev Mol Cell Biol 16, 651-664.

Bulkley, D., Innis, C. A., Blaha, G., and Steitz, T. A. (2010). Revisiting the structures of several antibiotics bound to the bacterial ribosome. Proc Natl Acad Sci U S A 107, 17158-17163.

Burkhardt, D. H., Rouskin, S., Zhang, Y., Li, G. W., Weissman, J. S., and Gross, C. A. (2017). Operon mRNAs are organized into ORF-centric structures that predict translation efficiency. Elife 6, e22037.

Byrgazov, K., Grishkovskaya, I., Arenz, S., Coudevylle, N., Temmel, H., Wilson, D. N., Djinovic-Carugo, K., and Moll, I. (2015). Structural basis for the interaction of protein S1 with the Escherichia coli ribosome. Nucleic Acids Res 43, 661-673.

Carter, A. P., Clemons, W. M., Brodersen, D. E., Morgan-Warren, R. J., Wimberly, B. T., and Ramakrishnan, V. (2000). Functional insights from the structure of the 30S ribosomal subunit and its interactions with antibiotics. Nature 407, 340-348.

Chadani, Y., Niwa, T., Chiba, S., Taguchi, H., and Ito, K. (2016). Integrated in vivo and in vitro nascent chain profiling reveals widespread translational pausing. Proc Natl Acad Sci U S A 113, E829-38.

Chadani, Y., Niwa, T., Izumi, T., Sugata, N., Nagao, A., Suzuki, T., Chiba, S., Ito, K., and Taguchi, H. (2017). Intrinsic ribosome destabilization underlies translation and 
provides an organism with a strategy of environmental sensing. Mol Cell 68, 528539.e5.

Charneski, C. A., and Hurst, L. D. (2013). Positively charged residues are the major determinants of ribosomal velocity. PLoS Biol 11, e1001508.

Chen, V. B., Arendall, W. B., Headd, J. J., Keedy, D. A., Immormino, R. M., Kapral, G. J., Murray, L. W., Richardson, J. S., and Richardson, D. C. (2010). MolProbity: allatom structure validation for macromolecular crystallography. Acta Crystallogr D Biol Crystallogr 66, 12-21.

Cornish, P. V., Ermolenko, D. N., Noller, H. F., and Ha, T. (2008). Spontaneous intersubunit rotation in single ribosomes. Mol Cell 30, 578-588.

Dana, A., and Tuller, T. (2012). Determinants of translation elongation speed and ribosomal profiling biases in mouse embryonic stem cells. PLoS Comput Biol 8 , e1002755.

Dao Duc, K., and Song, Y. S. (2018). The impact of ribosomal interference, codon usage, and exit tunnel interactions on translation elongation rate variation. PLoS Genet 14, e1007166.

Datsenko, K. A., and Wanner, B. L. (2000). One-step inactivation of chromosomal genes in Escherichia coli K-12 using PCR products. Proc Natl Acad Sci U S A 97, $6640-6645$.

Demo, G., Rasouly, A., Vasilyev, N., Svetlov, V., Loveland, A. B., Diaz-Avalos, R., Grigorieff, N., Nudler, E., and Korostelev, A. A. (2017). Structure of RNA polymerase bound to ribosomal 30S subunit. Elife 6, e28560 e28560. 
Diament, A., Feldman, A., Schochet, E., Kupiec, M., Arava, Y., and Tuller, T. (2018). The extent of ribosome queuing in budding yeast. PLoS Comput Biol 14, e1005951. Dunkle, J. A., Wang, L., Feldman, M. B., Pulk, A., Chen, V. B., Kapral, G. J., Noeske, J., Richardson, J. S., Blanchard, S. C., and Cate, J. H. (2011). Structures of the bacterial ribosome in classical and hybrid states of tRNA binding. Science 332, 981-984. Dunkle, J. A., Xiong, L., Mankin, A. S., and Cate, J. H. (2010). Structures of the Escherichia coli ribosome with antibiotics bound near the peptidyl transferase center explain spectra of drug action. Proc Natl Acad Sci U S A 107, 17152-17157.

Duval, M., Korepanov, A., Fuchsbauer, O., Fechter, P., Haller, A., Fabbretti, A., Choulier, L., Micura, R., Klaholz, B. P., Romby, P., Springer, M., and Marzi, S. (2013). Escherichia coli ribosomal protein $\mathrm{S} 1$ unfolds structured mRNAs onto the ribosome for active translation initiation. PLoS Biol 11, e1001731.

Emsley, P., Lohkamp, B., Scott, W. G., and Cowtan, K. (2010). Features and development of Coot. Acta Crystallogr D Biol Crystallogr 66, 486-501.

Ermolenko, D. N., Spiegel, P. C., Majumdar, Z. K., Hickerson, R. P., Clegg, R. M., and Noller, H. F. (2007). The antibiotic viomycin traps the ribosome in an intermediate state of translocation. Nat Struct Mol Biol 14, 493-497.

Fischer, N., Neumann, P., Konevega, A. L., Bock, L. V., Ficner, R., Rodnina, M. V., and Stark, H. (2015). Structure of the E. coli ribosome-EF-Tu complex at $<3 \AA$ resolution by Cs-corrected cryo-EM. Nature 520, 567-570.

Fujita, T., Kurihara, Y., and Iwasaki, S. (2019). The plant translatome surveyed by ribosome profiling. Plant Cell Physiol 60, 1917-1926.

Gagarinova, A., Stewart, G., Samanfar, B., Phanse, S., White, C. A., Aoki, H., Deineko, V., Beloglazova, N., Yakunin, A. F., Golshani, A., Brown, E. D., Babu, M., and Emili, 
A. (2016). Systematic genetic screens reveal the dynamic global functional organization of the bacterial translation machinery. Cell Rep 17, 904-916.

Garreau de Loubresse, N., Prokhorova, I., Holtkamp, W., Rodnina, M. V., Yusupova, G., and Yusupov, M. (2014). Structural basis for the inhibition of the eukaryotic ribosome. Nature 513, 517-522.

Goddard, T. D., Huang, C. C., Meng, E. C., Pettersen, E. F., Couch, G. S., Morris, J. H., and Ferrin, T. E. (2018). UCSF ChimeraX: Meeting modern challenges in visualization and analysis. Protein Sci 27, 14-25.

Gong, F., Ito, K., Nakamura, Y., and Yanofsky, C. (2001). The mechanism of tryptophan induction of tryptophanase operon expression: tryptophan inhibits release factor-mediated cleavage of TnaC-peptidyl-tRNA(Pro). Proc Natl Acad Sci U S A 98, $8997-9001$.

Gupta, R., Kasturi, P., Bracher, A., Loew, C., Zheng, M., Villella, A., Garza, D., Hartl, F. U., and Raychaudhuri, S. (2011). Firefly luciferase mutants as sensors of proteome stress. Nat Methods $8,879-884$.

Hussmann, J. A., Patchett, S., Johnson, A., Sawyer, S., and Press, W. H. (2015).

Understanding biases in ribosome profiling experiments reveals signatures of translation dynamics in yeast. PLoS Genet 11, e1005732.

Huter, P., Arenz, S., Bock, L. V., Graf, M., Frister, J. O., Heuer, A., Peil, L., Starosta, A. L., Wohlgemuth, I., Peske, F., Nováček, J., Berninghausen, O., Grubmüller, H., Tenson, T., Beckmann, R., Rodnina, M. V., Vaiana, A. C., and Wilson, D. N. (2017). Structural basis for polyproline-mediated ribosome stalling and rescue by the translation elongation factor EF-P. Mol Cell 68, 515-527.e6. 
Ibrahim, F., Maragkakis, M., Alexiou, P., and Mourelatos, Z. (2018). Ribothrypsis, a novel process of canonical mRNA decay, mediates ribosome-phased mRNA endonucleolysis. Nat Struct Mol Biol 25, 302-310.

Ingolia, N. T. (2014). Ribosome profiling: new views of translation, from single codons to genome scale. Nat Rev Genet 15, 205-213.

Ingolia, N. T., Brar, G. A., Rouskin, S., McGeachy, A. M., and Weissman, J. S. (2012). The ribosome profiling strategy for monitoring translation in vivo by deep sequencing of ribosome-protected mRNA fragments. Nat Protoc 7, 1534-1550.

Ingolia, N. T., Ghaemmaghami, S., Newman, J. R., and Weissman, J. S. (2009).

Genome-wide analysis in vivo of translation with nucleotide resolution using ribosome profiling. Science 324, 218-223.

Iwasaki, S., and Ingolia, N. T. (2017). The growing toolbox for protein synthesis studies. Trends Biochem Sci 42, 612-624.

Iwasaki, S., Iwasaki, W., Takahashi, M., Sakamoto, A., Watanabe, C., Shichino, Y., Floor, S. N., Fujiwara, K., Mito, M., Dodo, K., Sodeoka, M., Imataka, H., Honma, T., Fukuzawa, K., Ito, T., and Ingolia, N. T. (2019). The translation Inhibitor rocaglamide targets a bimolecular cavity between eIF4A and polypurine RNA. Mol Cell 73, 738748.e9.

Jenner, L., Starosta, A. L., Terry, D. S., Mikolajka, A., Filonava, L., Yusupov, M., Blanchard, S. C., Wilson, D. N., and Yusupova, G. (2013). Structural basis for potent inhibitory activity of the antibiotic tigecycline during protein synthesis. Proc Natl Acad Sci U S A 110, 3812-3816.

Kaminishi, T., Wilson, D. N., Takemoto, C., Harms, J. M., Kawazoe, M., Schluenzen, F., Hanawa-Suetsugu, K., Shirouzu, M., Fucini, P., and Yokoyama, S. (2007). A 
snapshot of the 30S ribosomal subunit capturing mRNA via the Shine-Dalgarno interaction. Structure 15, 289-297.

Kitagawa, M., Ara, T., Arifuzzaman, M., Ioka-Nakamichi, T., Inamoto, E., Toyonaga, H., and Mori, H. (2005). Complete set of ORF clones of Escherichia coli ASKA library (a complete set of $E$. coli K-12 ORF archive): unique resources for biological research. DNA Res 12, 291-299.

Kurihara, Y., Makita, Y., Kawashima, M., Fujita, T., Iwasaki, S., and Matsui, M. (2018). Transcripts from downstream alternative transcription start sites evade uORFmediated inhibition of gene expression in. Proc Natl Acad Sci U S A 115, 7831-7836. Lareau, L. F., Hite, D. H., Hogan, G. J., and Brown, P. O. (2014). Distinct stages of the translation elongation cycle revealed by sequencing ribosome-protected mRNA fragments. Elife 3, e01257.

Lu, J., and Deutsch, C. (2008). Electrostatics in the ribosomal tunnel modulate chain elongation rates. J Mol Biol 384, 73-86.

Mastronarde, D. N. (2005). Automated electron microscope tomography using robust prediction of specimen movements. J Struct Biol 152, 36-51.

Matsuo, Y., Ikeuchi, K., Saeki, Y., Iwasaki, S., Schmidt, C., Udagawa, T., Sato, F., Tsuchiya, H., Becker, T., Tanaka, K., Ingolia, N. T., Beckmann, R., and Inada, T. (2017). Ubiquitination of stalled ribosome triggers ribosome-associated quality control. Nat Commun 8, 159.

McGlincy, N. J., and Ingolia, N. T. (2017). Transcriptome-wide measurement of translation by ribosome profiling. Methods 126, 112-129. 
Mohammad, F., Green, R., and Buskirk, A. R. (2019). A systematically-revised ribosome profiling method for bacteria reveals pauses at single-codon resolution. Elife $8, \mathrm{e} 42591$.

Mohammad, F., Woolstenhulme, C. J., Green, R., and Buskirk, A. R. (2016). Clarifying the translational pausing landscape in bacteria by ribosome profiling. Cell Rep 14, 686694.

Muto, H., Nakatogawa, H., and Ito, K. (2006). Genetically encoded but nonpolypeptide prolyl-tRNA functions in the A site for SecM-mediated ribosomal stall. Mol Cell 22, $545-552$.

Nakatogawa, H., and Ito, K. (2001). Secretion monitor, SecM, undergoes selftranslation arrest in the cytosol. Mol Cell 7, 185-192.

Nakatogawa, H., and Ito, K. (2002). The ribosomal exit tunnel functions as a discriminating gate. Cell 108, 629-636.

Pettersen, E. F., Goddard, T. D., Huang, C. C., Couch, G. S., Greenblatt, D. M., Meng, E. C., and Ferrin, T. E. (2004). UCSF Chimera--a visualization system for exploratory research and analysis. J Comput Chem 25, 1605-1612.

Pop, C., Rouskin, S., Ingolia, N. T., Han, L., Phizicky, E. M., Weissman, J. S., and Koller, D. (2014). Causal signals between codon bias, mRNA structure, and the efficiency of translation and elongation. Mol Syst Biol 10, 770.

Qu, X., Lancaster, L., Noller, H. F., Bustamante, C., and Tinoco, I. (2012). Ribosomal protein S1 unwinds double-stranded RNA in multiple steps. Proc Natl Acad Sci U S A $109,14458-14463$.

Rohou, A., and Grigorieff, N. (2015). CTFFIND4: Fast and accurate defocus estimation from electron micrographs. J Struct Biol 192, 216-221. 
Sato, K., Hamada, M., Asai, K., and Mituyama, T. (2009). CENTROIDFOLD: a web server for RNA secondary structure prediction. Nucleic Acids Res 37, W277-80.

Schneider-Poetsch, T., Ju, J., Eyler, D. E., Dang, Y., Bhat, S., Merrick, W. C., Green, R., Shen, B., and Liu, J. O. (2010). Inhibition of eukaryotic translation elongation by cycloheximide and lactimidomycin. Nat Chem Biol 6, 209-217.

Schuller, A. P., Wu, C. C., Dever, T. E., Buskirk, A. R., and Green, R. (2017). eIF5A functions globally in translation elongation and termination. Mol Cell 66, 194-205.e5. Sengupta, J., Agrawal, R. K., and Frank, J. (2001). Visualization of protein S1 within the 30S ribosomal subunit and its interaction with messenger RNA. Proc Natl Acad Sci U S A 98, 11991-11996.

Sharma, A. K., Sormanni, P., Ahmed, N., Ciryam, P., Friedrich, U. A., Kramer, G., and O’Brien, E. P. (2019). A chemical kinetic basis for measuring translation initiation and elongation rates from ribosome profiling data. PLoS Comput Biol 15, e1007070. Shimizu, Y., Inoue, A., Tomari, Y., Suzuki, T., Yokogawa, T., Nishikawa, K., and Ueda, T. (2001). Cell-free translation reconstituted with purified components. Nat Biotechnol 19, 751-755.

Stanley, R. E., Blaha, G., Grodzicki, R. L., Strickler, M. D., and Steitz, T. A. (2010). The structures of the anti-tuberculosis antibiotics viomycin and capreomycin bound to the 70S ribosome. Nat Struct Mol Biol 17, 289-293.

Subramaniam, A. R., Zid, B. M., and O’Shea, E. K. (2014). An integrated approach reveals regulatory controls on bacterial translation elongation. Cell 159, 1200-1211. Svidritskiy, E., Ling, C., Ermolenko, D. N., and Korostelev, A. A. (2013). Blasticidin S inhibits translation by trapping deformed tRNA on the ribosome. Proc Natl Acad Sci U S A $110,12283-12288$. 
Voorhees, R. M., and Ramakrishnan, V. (2013). Structural basis of the translational elongation cycle. Annu Rev Biochem 82, 203-236.

Wang, L., Pulk, A., Wasserman, M. R., Feldman, M. B., Altman, R. B., Cate, J. H., and Blanchard, S. C. (2012). Allosteric control of the ribosome by small-molecule antibiotics. Nat Struct Mol Biol 19, 957-963.

Weinberg, D. E., Shah, P., Eichhorn, S. W., Hussmann, J. A., Plotkin, J. B., and Bartel, D. P. (2016). Improved ribosome-footprint and mRNA measurements provide insights into dynamics and regulation of yeast translation. Cell Rep 14, 1787-1799.

Wilson, D. N., Schluenzen, F., Harms, J. M., Starosta, A. L., Connell, S. R., and Fucini, P. (2008). The oxazolidinone antibiotics perturb the ribosomal peptidyl-transferase center and effect tRNA positioning. Proc Natl Acad Sci U S A 105, 13339-13344. Woolstenhulme, C. J., Guydosh, N. R., Green, R., and Buskirk, A. R. (2015). Highprecision analysis of translational pausing by ribosome profiling in bacteria lacking EFP. Cell Rep 11, 13-21.

Wu, C. C., Zinshteyn, B., Wehner, K. A., and Green, R. (2019). High-resolution ribosome profiling defines discrete ribosome elongation states and translational regulation during cellular stress. Mol Cell 73, 959-970.e5.

Yanagitani, K., Kimata, Y., Kadokura, H., and Kohno, K. (2011). Translational pausing ensures membrane targeting and cytoplasmic splicing of XBP1u mRNA. Science 331, 586-589.

Zarnegar, B. J., Flynn, R. A., Shen, Y., Do, B. T., Chang, H. Y., and Khavari, P. A. (2016). irCLIP platform for efficient characterization of protein-RNA interactions. Nat Methods 13, 489-492. 
Zhang, J., Pan, X., Yan, K., Sun, S., Gao, N., and Sui, S. F. (2015). Mechanisms of ribosome stalling by SecM at multiple elongation steps. Elife 4, e09684.

Zhang, S., Hu, H., Zhou, J., He, X., Jiang, T., and Zeng, J. (2017). Analysis of ribosome stalling and translation elongation dynamics by deep learning. Cell Syst 5, 212-220.e6. Zivanov, J., Nakane, T., Forsberg, B. O., Kimanius, D., Hagen, W. J., Lindahl, E., and Scheres, S. H. (2018). New tools for automated high-resolution cryo-EM structure determination in RELION-3. Elife 7, 


\section{Figures}

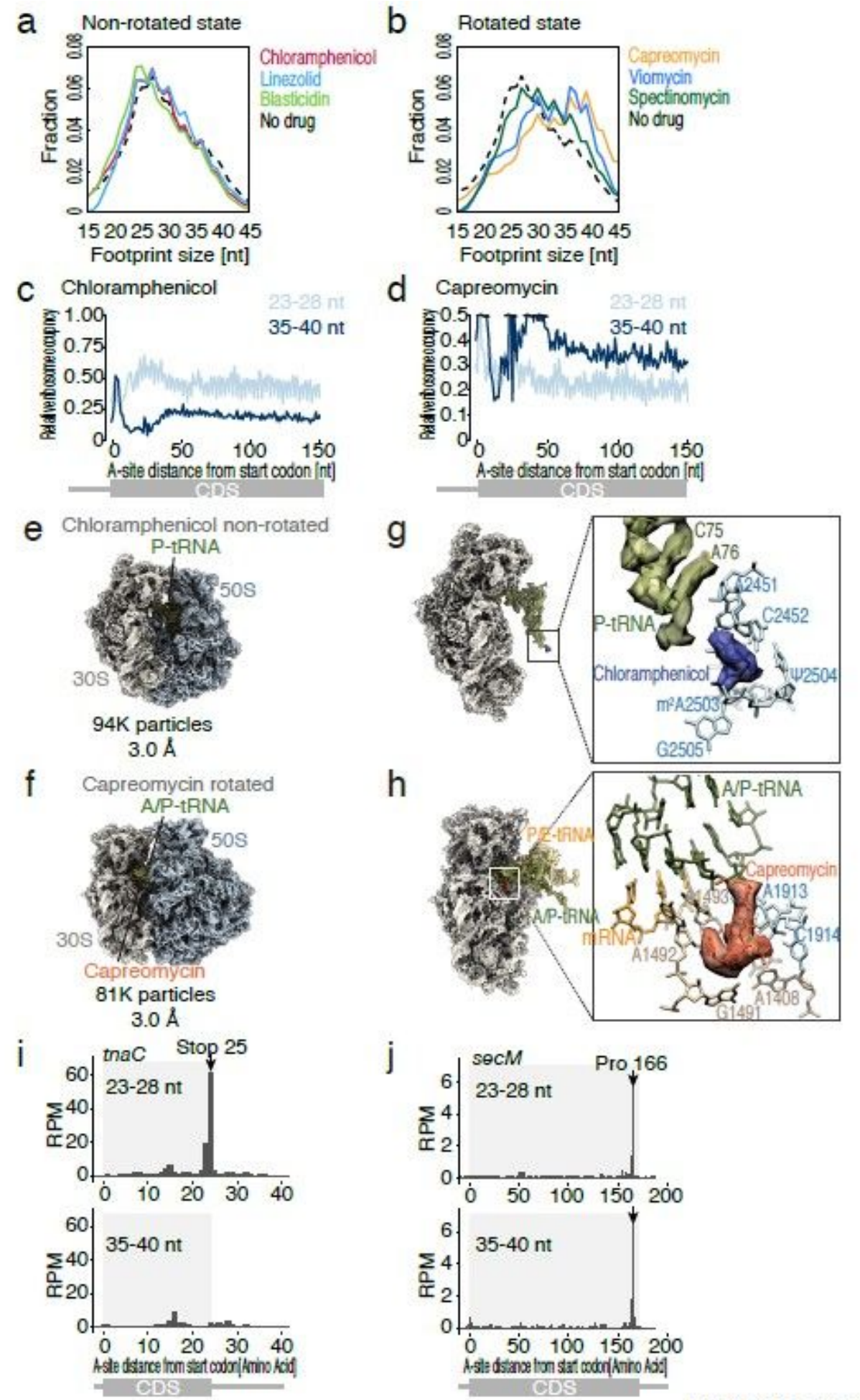

Fig. 1 Fujita et al. 2020

\section{Figure 1}

Differential sizes of ribosome-protected fragments generated by translation inhibitors. ( $a$ and b) Footprint size distributions in ribosome profiling with peptidyl transfer inhibitors (a) and translocation inhibitors (b). (c and d). Normalized ribosome occupancy of the indicated size of footprints with chloramphenicol (c) 
and capreomycin (d) treatments around the start codon. (e and f) Ribosome conformation revealed by cryo-EM under chloramphenicol (e) and capreomycin (f) treatments. (g) A close-up view of chloramphenicol binding to PTC shown with the ribosomal environment. 50S subunit is removed to show the details. (h) A close-up view of capreomycin binding to the $16 \mathrm{~S}$ decoding center shown with the ribosomal environment. 50S subunit is removed to show the details. ( $i$ and $j$ ) The footprint distribution at the A-site position of reads along tnaC (i) and secM (j). The indicated sizes of footprints are depicted. The known pause sites are indicated by arrows. RPM: reads per million mapped reads
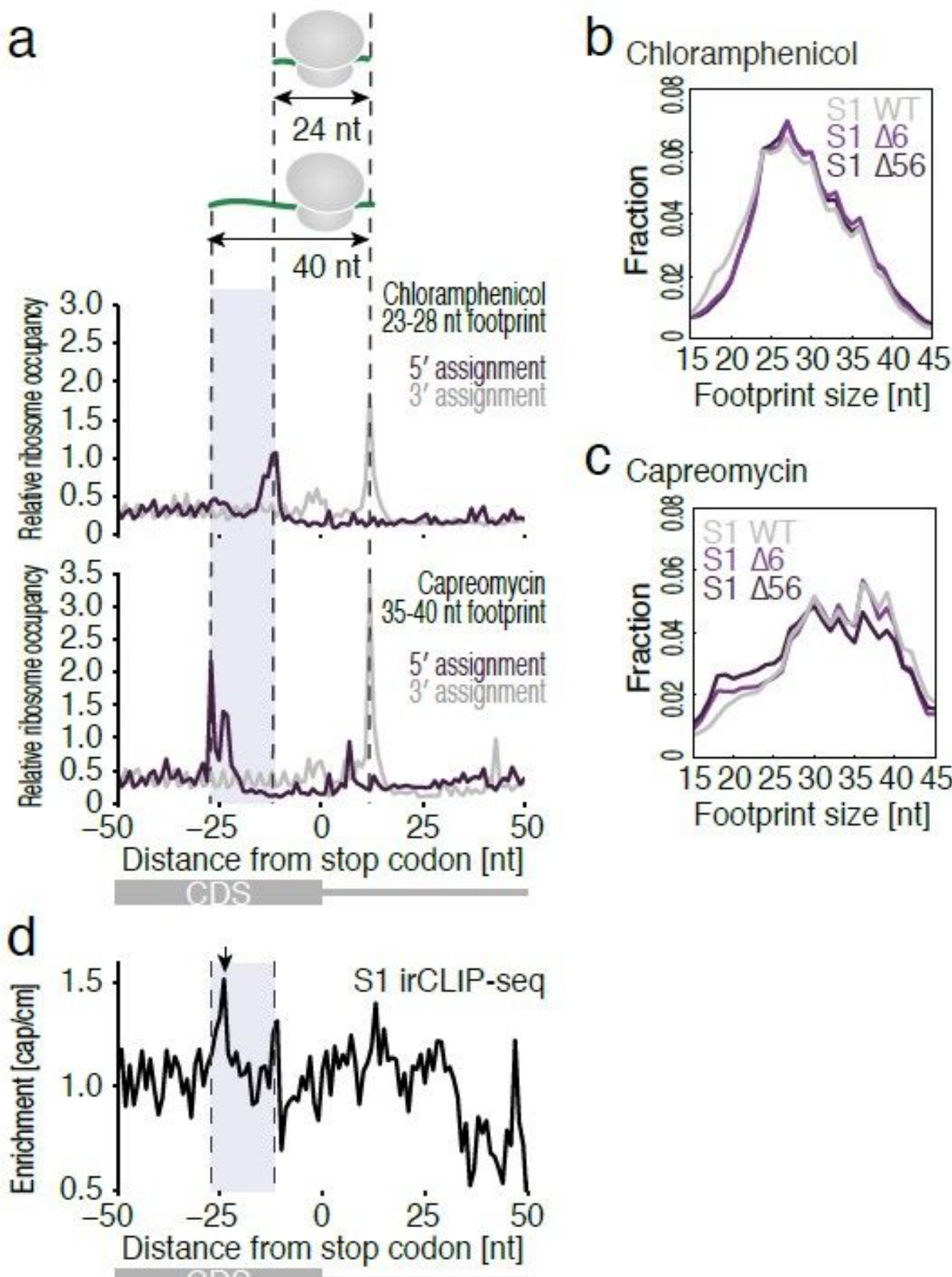

C Capreomycin

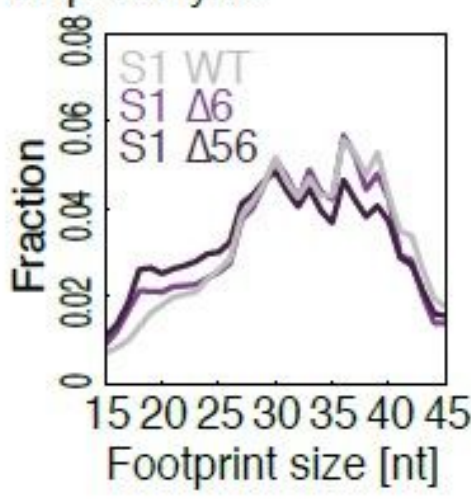

Distance from stop codon [nt] 
Figure 2

S1 protein extends footprints in the rotated state of ribosomes. (a) Meta-gene analysis showing the $5^{\prime}$ and 3 ' ends of footprints around stop codons. Short (23-28 nt) (top) and long (35-40 nt) (bottom) chloramphenicol- and capreomycin-treated samples, respectively, are shown. (b and c) Footprint size distributions from S1 WT, S1 $\Delta 6$, and S1 $\Delta 56$ strains with chloramphenicol (b) and with capreomycin (c). (d) Read enrichment of S1 irCLIP with capreomycin (cap) over chloramphenicol (cm) treatment, around stop codon. The $5^{\prime}$ end of reads is shown. The enriched site, which is corresponded to the footprint extension at the stop codon, is indicated by black arrow.

a

\section{b}

CDS length [codon]

I $\square<150$

II $\square 151-300$

III $\square 301-450$

IV $\square>451$

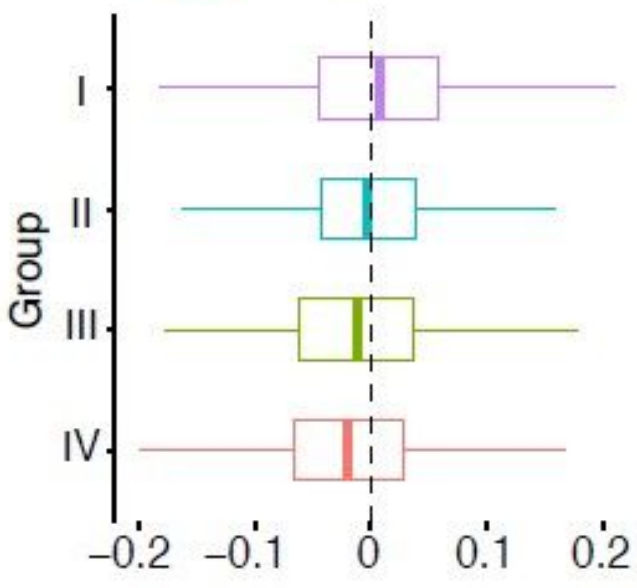

$\Delta$ Polarity score [S1 $\Delta 56$ vs. S1 WT] $5^{\prime}$

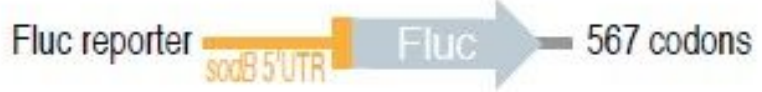

1xGFP-Fluc reporter $\frac{\text { SodB 5UTR }}{\text { GEFP. Fluc }}=816$ codons

2xGFP-Fluc reporter

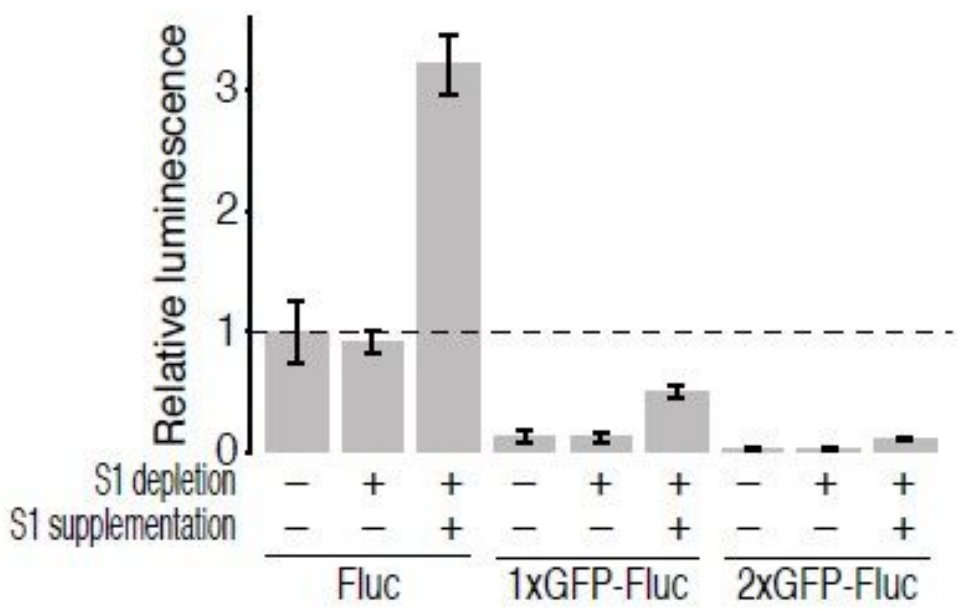

\section{Figure 3}

S1 facilitates translation elongation from long CDS. (a) The difference of polarity scores of footprints in S1 $\Delta 56$ strain compared S1 WT strain. The mRNAs were sub-classed into four groups according to the CDS length. (b) Schematic representation of reporters constructed (top). sodB 5' UTR, which enables S1independent translation initiation, is fused to firefly luciferase (Fluc). Single or tandem GFPs were inserted to extend CDS length. These reporters were translated by S1-depleted ribosomes with in vitro translation system (bottom). Data represent mean and S.D. $(n=3)$. 


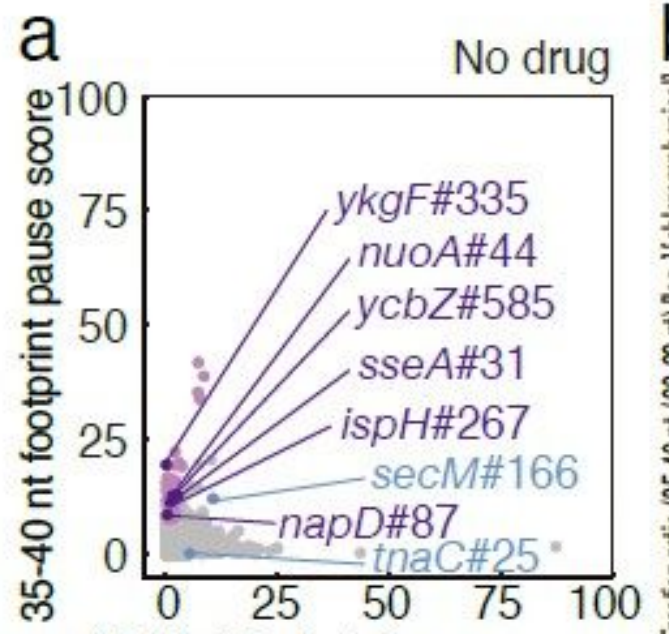

23-28 nt footprint pause score

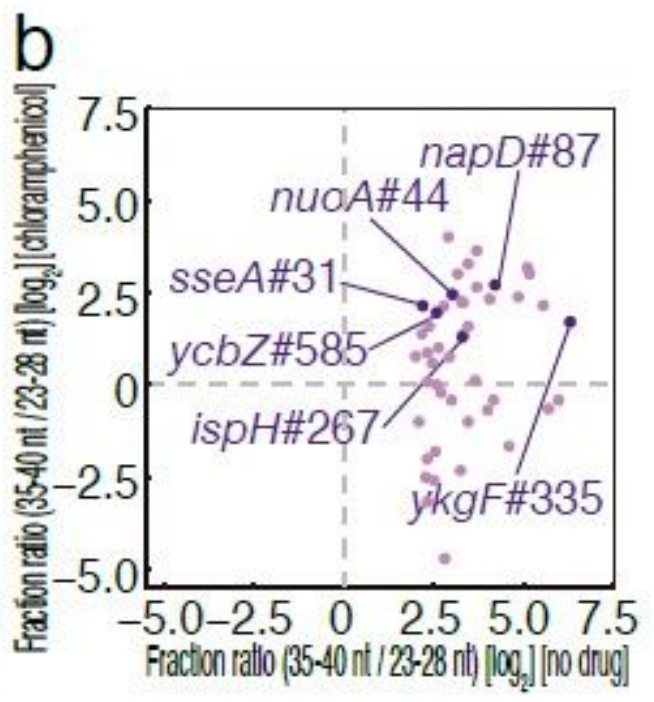

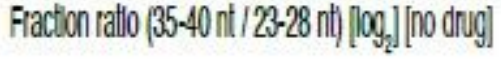

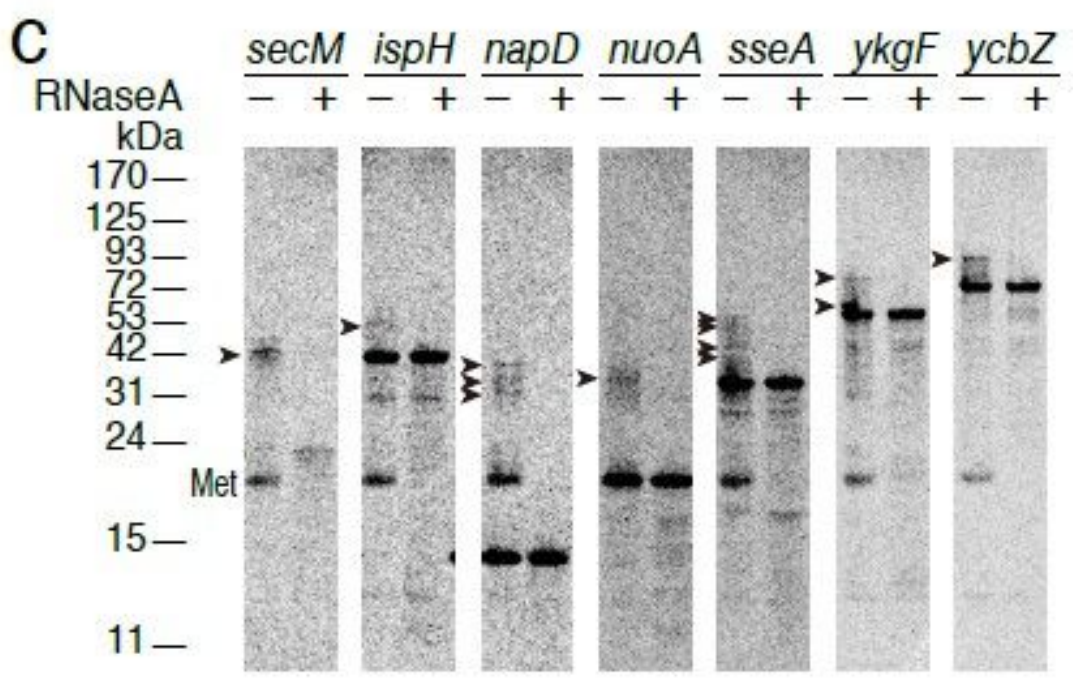

\section{Figure 4}

ycbZ mRNA has a ribosome pause site with long footprints. (a) Scatterplot representing long footprint (35-40 nt) versus short footprint (23-27 nt) accumulation across all codons of the transcriptome without drug treatment. Light purple: codons with long footprint over-represented, which are defined as long footprint pause scores larger than mean +11.5 S.D. and a ratio of long footprint to short footprint larger than 0.8; blue: sites tested for iNP in (c), and dark purple: known arrest sites in secM and tnaC mRNAs. \# and following number stand for A-site position of codon. (b) Scatterplot representing the ratio of long footprint (35-40 nt) to short footprint (23-27nt) at the selected codon positions in drug-free and chloramphenicol-treated conditions. (c) iNP for selected mRNAs highlighted in (a) and (b). RNasesensitive peptidyl-tRNAs are indicated by arrowheads. 


\section{a}
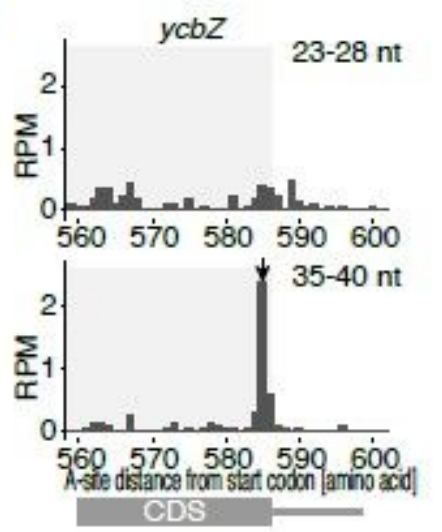

b

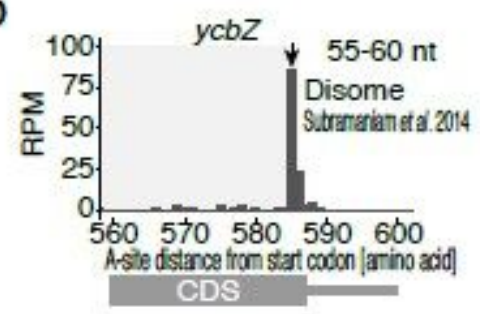

g

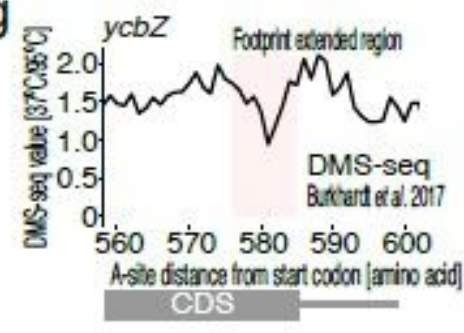

e

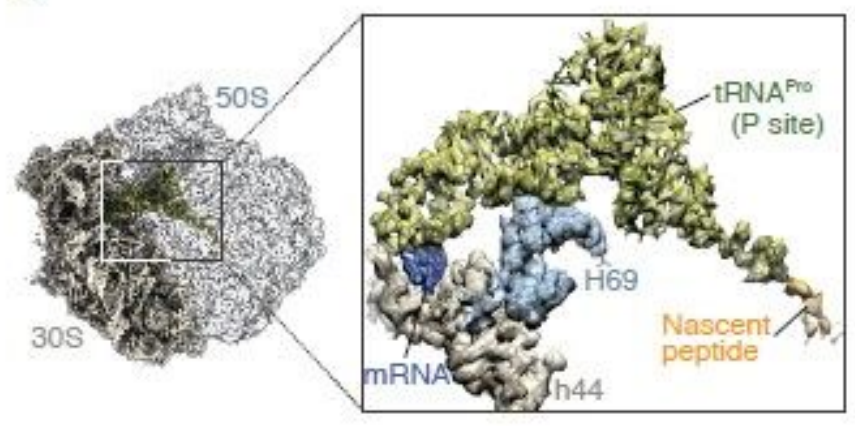

C

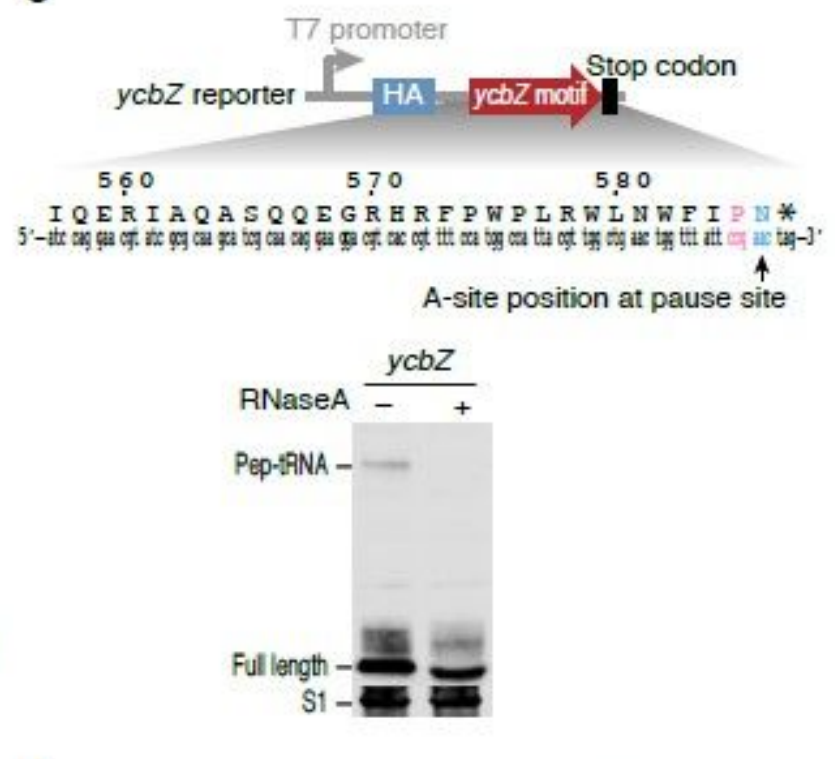

d

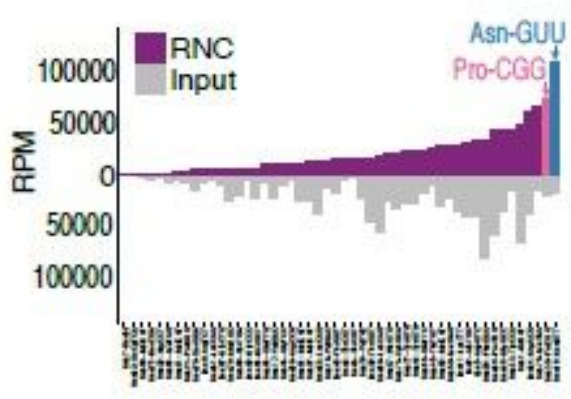

f

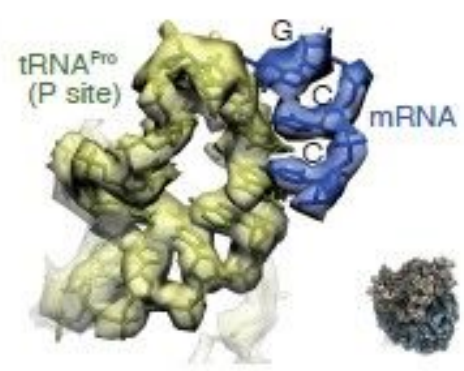

\section{Figure 5}

Ribosomes stalled at the ycbZ pause site revealed by cryo-EM. (a) The footprint distribution at the A-site position of reads along ycbZ. The indicated sizes of footprints are depicted. The tested pause sites are indicated by an arrow. (b) Same as (a) but for disome footprints reported in (Subramaniam et al., 2014). (c) Schematic representation of reporter constructed (top) and Western blot for peptidyltRNA (probed by HA epitope) from the reporter run in SDS-PAGE under natural $\mathrm{pH}$ (bottom). RNase treatment verified the RNA conjugation. (d) Enrichment of tRNAs in the RNC at the ycbZ pause site, assessed by tRNA-seq. (e and f) Close-up view of the inter-subunit cavity of this complex depicting the structural details of the 
nascent peptide-bound P-site tRNA (e). The structural model of tRNAProCGG is well fitted to the corresponding cryo-EM density. The details of the codon-anticodon recognition of P-site tRNA are shown in (f). (g) RNA base-paring around the ycbZ pause site (indicated by an arrow), probed by DMSseq (Burkhardt et al., 2017). Reads in a $33 \mathrm{nt}$ window were summed and normalized. Red shade shows footprint extended region upstream of A site.

\section{Short footprint}

\section{Long footprint}

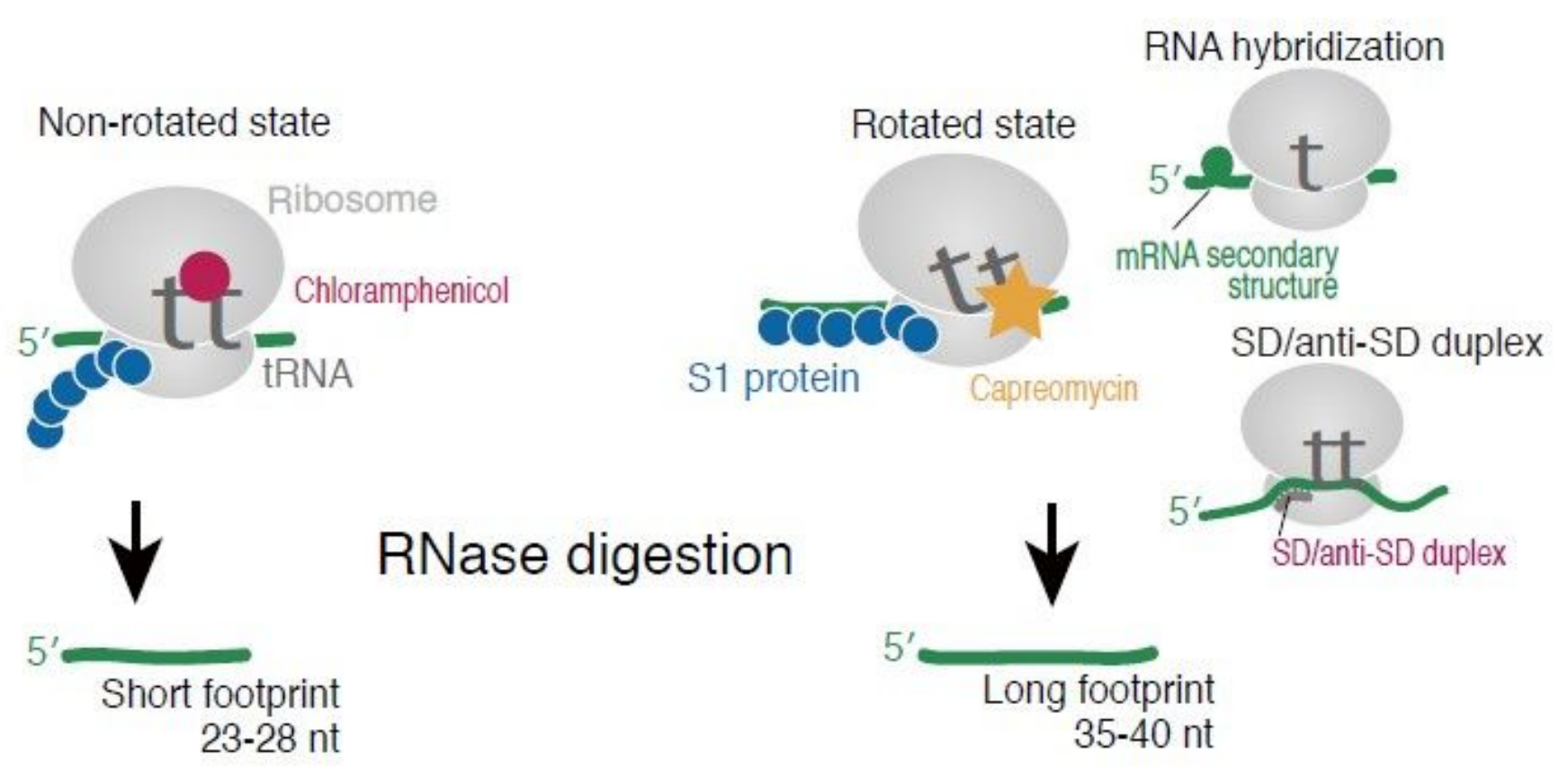

Figure 6

Schematic representation of bacterial footprint extension. During bacterial ribosome profiling, short footprints (23-27 nt) are originated from nonrotated ribosomes, which could be trapped by chloramphenicol. Long footprints (35-40nt) are generated by subunit rotation. Rotated ribosome (stabilized by capreomycin) leads to high probability of mRNA association by $\mathrm{S} 1$, which sterically inhibits a RNase attack. A minor fraction of long footprints are originated by intra-molecular and inter-molecular (SD/andi-SD) base-paring, that are resistant to RNase digestion.

\section{Supplementary Files}

This is a list of supplementary files associated with this preprint. Click to download.

- FigureS1Fujita.jpg

- FigureS2Fujita.jpg 
- FigureS3Fujita.jpg

- FigureS4Fujita.jpg

- FigureS5Fujita.jpg

- FigureS6Fujita.jpg 